\title{
Improving Rehabilitation Patient's Performance in Exercise Games using Collaborative Filtering Approach
}

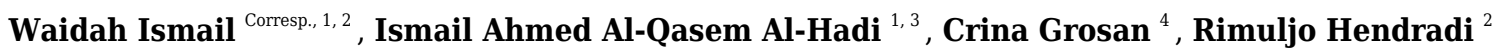 \\ 1 Faculty of Science and Technology, Universiti Sains Islam Malaysia, Nilai, Negeri Sembilan, Malaysia \\ 2 Information System Study Program, Universitas Airlangga, Indonesia Kampus C, Surabaya, Indonesia \\ 3 Faculty of Ocean Engineering Technology and Informatics, Universiti Malaysia Terengganu, Kuala Nerus, Terengganu, Malaysia \\ 4 Dept of Computer Science, Brunel University, London, United Kingdom \\ Corresponding Author: Waidah Ismail \\ Email address: waidah@usim.edu.my
}

Background. Virtual reality is utilised in exergames to help patients with disabilities improve on the movement of their limbs. Exergame settings, such as the game difficulty, play important roles in the rehabilitation outcome. Similarly, suboptimal exergames' settings may adversely affect the accuracy of the results obtained. As such, the improvement in patients' movement performances falls below the desired expectations. In this paper, the recommender system is incorporated to suggest the most preferred movement setting for each patient, based on the movement history of the patient. Method. The proposed recommender system (ResComS) suggests the most suitable setting necessary to optimally improve patients' rehabilitation performances. In the course of developing the recommender system, three methods are proposed and compared: ReComS (K-nearest neighbours and collaborative filtering algorithms), ReComS+ ( $k$ means, K-nearest neighbours, and collaborative filtering algorithms) and ReComS++ (bacterial foraging optimisation, $k$-means, K-nearest neighbours, and collaborative filtering algorithms). The experimental datasets are collected using the Medical Interactive Recovery Assistant (MIRA) software platform. Result. Experimental results, validated by the patients' exergame performances, reveal that the ReComS ++ approach predicts the best exergame settings for patients with $85.76 \%$ accuracy prediction. 
1 Improving Rehabilitation Patient's Performance in

2 Exercise Games using Collaborative Filtering

3 Approach

5 Waidah Ismail ${ }^{1,2}$, Ismail Ahmed Al-Qasem Al-Hadi ${ }^{1,3}$, Crina Grosan ${ }^{4}$, Rimuljo

6 Hendradi $^{2}$

7

8

${ }^{1}$ Faculty of Science and Technology, Universiti Sains Islam Malaysia, Nilai, Negeri Sembilan, Malaysia

${ }^{2}$ Information System Study Program, Universitas Airlangga, Indonesia Kampus C, Surabaya, Indonesia.

${ }^{3}$ Faculty of Ocean Engineering Technology and Informatics, Universiti Malaysia Terengganu, Kuala Nerus, Terengganu, Malaysia

${ }^{4}$ Dept of Computer Science, Brunel University, London, United Kingdom.

Corresponding Author:

Waidah Ismail, waidah@usim.edu.my

\section{Abstract}

Background. Virtual reality is utilised in exergames to help patients with disabilities improve on the movement of their limbs. Exergame settings, such as the game difficulty, play important roles in the rehabilitation outcome. Similarly, suboptimal exergames' settings may adversely affect the accuracy of the results obtained. As such, the improvement in patients' movement performances falls below the desired expectations. In this paper, the recommender system is incorporated to suggest the most preferred movement setting for each patient, based on the movement history of the patient.

Method. The proposed recommender system (ResComS) suggests the most suitable setting necessary to optimally improve patients' rehabilitation performances. In the course of developing the recommender system, three methods are proposed and compared: ReComS (K-nearest neighbours and collaborative filtering algorithms), ReComS $+(k$-means, $\mathrm{K}$-nearest neighbours, and collaborative filtering algorithms) and $\mathrm{ReComS++}$ (bacterial foraging optimisation, $k$-means, $\mathrm{K}$ nearest neighbours, and collaborative filtering algorithms). The experimental datasets are collected using the Medical Interactive Recovery Assistant (MIRA) software platform.

Result. Experimental results, validated by the patients' exergame performances, reveal that the ReComS++ approach predicts the best exergame settings for patients with $85.76 \%$ accuracy prediction. 
39

40

41

42

43

44

45

46

47

48

49

50

51

52

53

54

55

56

57

58

59

60

61

62

63

64

65

66

67

68

69

70

71

72

73

74

75

76

77

78

79

80

81

82

\section{Introduction}

Human disabilities could develop through cerebral palsy (CP), stroke, spinal cord injury (SCI), traumatic brain injury (TBI), and humerus fracture (Tousignant et al., 2014). Rehabilitating these disabled patients can be achieved using traditional and modern treatments. Virtual reality (Turolla et al., 2013), robotics (Díaz, Gil \& Sánchez, 2011) (Maciejasz et al., 2014), simulation, and exergames (Covarrubias et al., 2015) are commonly used in modern treatments. These games are quite promising as they furnish patients with new experiences while performing their daily exercises which are rehabilitation therapies (Li et al., 2018a). Rehabilitation is traditionally based on the assessment requirements drawn through physiotherapy. Rehabilitation therapy and assessment are provided by rehabilitation centres, where patients train their disabled limbs through a series of pre-determined exercises. Such procedures help to improve the movement of the limbs and improve their functionality.

One of the recently developed systems for training lower limbs is the robotic system. Currently, there are five types: foot-plate-based gait trainers, treadmill gait trainers, overground gait trainers, active foot orthoses, in addition to stationary gait and ankle trainers (Díaz, Gil \& Sánchez, 2011). These systems include passive robotic devices, which assist the patient to train the idle $\operatorname{limb}(\mathrm{s})$ of the lower part. The virtual reality therapy (VRT) systems involve the use of virtual reality as assistant-tools for the rehabilitation process. In assistive technology, serious games come with multimodal functions and immersive characteristics that have been embedded into various types such as robotics, virtual reality, and simulator. Thus, serious games are promising technology that can bring new experiences for people with disabilities to perform their rehabilitation (Li et al., 2018b) (Merilampi et al., 2017). In addition, exergames are VRT systems that are used by patients who suffer from movement disability in their idle limbs. Through several training procedures, exergames help patients to improve on the physical movements of their muscles (Jaarsma et al., 2020) so that they can move their idle parts gradually. For example, the VRT system employs three exergames (the bike, the pedal boat, and the swimmer) that consists of a virtual system comprising a strengthening machine, Kinect device, large screen, and a computer (Pruna et al., 2018). As for the spine, the VRT system is a non-invasive alternative having minimal negative or harmful effects (Chi et al., 2019). The VRT systems for lower limbs and spine; however, remain inefficient as existing VRT systems only cater to the upper limbs.

Exergame therapy forms part of the rehabilitation approaches that are offered to patients in rehabilitation centres. Exergames refer to video games (Da Gama et al., 2016) that encourage patients to continue their exercise without feeling bored. The therapy consists of an iteration of exercises that focus mainly on strengthening a part of the patient's body such as the knee. For effective results, it is essential that the patient performs the right movement following the rules of each exergame; otherwise, the benefits may not be pronounced and the desired results will be less noticeable (Da Gama et al., 2016). Different devices provide the virtual environment based on the requirements of the VRT application. These include a large monitor, virtual interface (Brokaw \& Brewer, 2013), Microsoft Kinect, Xbox (Baur et al., 2018), strengthening machine (Pruna et al., 2018), customised metal rig that holds standard wheelchair and robotic devices (Radman, Ismail \& Bahari, 2018).

The Medical Interactive Recovery Assistant (MIRA) platform is a new VRT application that presents a wide variety of games and movements for various rehabilitation needs. It consists of three parts: adapted movement-based interactive video games, the Kinect, and the leap motion

Peer) Comput. Sci. reviewing PDF | (CS-2020:10:53958:2:0:NEW 11 May 2021) 
sensors (Moldovan et al., 2017). The Kinect sensor tracks motion and provides different interactions between the patient and the different types of exergames (Mcglinchey et al., 2015). Leap motion tracks the hand's movement that composed with the flexion gauges placed into the glove (Borja et al., 2018). In other words, the exergames in MIRA are created specifically to aid physical rehabilitation therapies and assessments. An example is that studies the movement performance of children who are seven years old and suffer from the brachial plexus palsy caused by transverse myelitis (Czakó, Silaghi \& Vizitiu, 2017). In the study, the movement performance of children was improved by MIRA exergames training. Another case study demonstrates that MIRA exergames have positive effects and can be safely implemented for adult patients (Mcglinchey et al., 2015). However, in both case studies (i.e., (Mcglinchey et al., 2015) and (Czakó, Silaghi \& Vizitiu, 2017)), a physiotherapist uses default exergames settings and no automation was considered. Prediction scoring method is used to suggest the comfortable difficulty mode for rehabilitation patients using k-means algorithm (Zainal et al., 2019). It used to analysis five variables that are generated by patients when playing MIRA exergames. However, there are various exergames with several variables need to analysis for finding the accurate prediction scoring.

In the above scenarios, the most suitable exergame settings are required considering each patient's disability type. As such, due to the low engagement between physiotherapists and patients, physiotherapists use default settings which invariably lowers the accuracy in playing the exergames and reduces the patients' performances. To overcome this problem, recommender system (RS) is needed in the MIRA platform. However, to our best, addressing this problem using $\mathrm{RS}$ is absent in the literature. In addition, the decision tree model applied to predict a patient's rehabilitation future performance is based on time, average acceleration, distance, moving time, and average speed. This prediction method uses the default exergame settings and the previous performances of patients who played the same exergame with the same side (Zainal et al., 2020). In spite of that, the prediction will be more accurate if the exergame settings are controlled automatically. Therefore, RS is utilised in this paper to suggest appropriate settings for patients who use MIRA to enhance their movement disabilities.

$\mathrm{RS}$ is a subcategory of an information filtering system that aims to forecast or project the "rating" or "preference" of a person (Ismail et al., 2019). Over the last ten years, RSs have been explored and used in various applications that include e-health, e-learning, e-commerce, and knowledge management systems (Xu, Zhang \& Yan, 2018) (Zainal et al., 2020). In the same manner, we deploy the RS in this research. Traditionally, an exergame records a patient's information and performance during a session. The recorded data is used to monitor the progress of the patient (Covarrubias et al., 2015). Likewise, the system analyses patients' movements during the exercise and generates statistical data. Nonetheless, several challenges have been identified while creating tailored exergame schedules for patients. In this respect, this paper explores the use of ReComS as an interface for each exergame, using the patient's movement history as a benchmark. To address the problem related to the input setting, the ReComS approach applies $k$ means, K-nearest neighbours $(\mathrm{K}-\mathrm{NN})$, collaborative filtering $(\mathrm{CF})$, and bacterial foraging optimisation algorithm (BFOA) to accurately predict input variables through an item settings dialogue box in the MIRA platform itself. From the above discussion, the primary contributions of this research include the following:

1. A proposed ReComS approach that suggests the most appropriate setting for enhancing rehabilitation patients' performances. 
2. A novel deployment of RS in the MIRA platform to accurately suggest the most suitable settings needed to improve the limb movement of patients.

3. An enhancement of input variables' prediction accuracy using three newly-developed comparison methods named ReComS (K-NN and CF algorithms), ReComS+ (k-means, K$\mathrm{NN}$ and $\mathrm{CF}$ algorithms) and $\mathrm{ReComS}++$ (BFOA, $k$-means, $\mathrm{K}-\mathrm{NN}$, and $\mathrm{CF}$ algorithms).

The remainder of this paper is structured as follows: Section 2 describes the MIRA platform. The concepts used in this paper are defined in Section 3. In Section 4, the proposed methods are provided. Sections 5 and 6 present the empirical results and conclusion, respectively.

\section{The MIRA Platform}

The MIRA platform is an effective system that allows patients to play their way towards recovery (Zainal et al., 2019). MIRA is a non-immersive type of VRT application developed to make physiotherapy entertaining and enjoyable for patients. The platform transforms prevailing physical therapy exercises into clinically-designed video games. Asides from improving patients' interests in exercising, an external sensor monitors and evaluates their adherence. MIRA contains a broad range of games and exercises for the upper limbs, lower limbs, and spine. Fig. 1 illustrates three instances of games in the MIRA platform. First, the patient plays the Catch game with hip abduction movement (Fig. 1(a)). In the second image, the patient plays the Airplane game with elbow flexion in abduction movement (Fig. 1(b)). In the third example, the patient plays the Flight control game with general shoulder movement (Fig. 1(c)). All MIRA games are played following the rules of each game and movement.

Kinects and screens are connected to computers (as shown in Fig. 1) to provide the virtual environment. The physiotherapist utilises these devices and the MIRA platform to create a session for patients based on their ability and rehabilitation needs. He combines exercises and games with the specific difficulty setting, movement tolerance, and range of movement (Wilson et al., 2017) based on his observations from the previous data of each patient. Through these settings, this research examines the input and output attributes of the scheduled games in order to autodetermine input variables for each exergame. Traditionally, the physiotherapist tracks the exergame history of each patient (that reflects the movement threshold of the idle limb) then suggests the values of input settings in the dialogue box for future exergaming. This method of observation is costly and time-consuming because the physiotherapist needs to prepare a manual list of variables to track the patient's history. Thus, most physiotherapists use the default setting for all patients which retards the patients' performances, especially when they play using their idle limbs. In view of the aforementioned, this research proposes ReComS approaches for learning the "best" setting for each patient. It deploys $k$-means, K-NN, and BFOA algorithms, in addition to the $\mathrm{CF}$ technique. The main reasons for using MIRA data include:

- MIRA data contains several exergame features that can be performed by moving the upper or lower limbs.

- Patients' data are normalized and arranged in a matrix of features. However, this matrix includes a high percentage of zeros (i.e., unknown values) because the exergame output variables are different due to patients' diverse movement skills.

- Some exergames generate a small number of records because only a few patients are interested in playing them.

Our approach is discussed further in the following subsections. 


\section{Definition of Concepts}

172

173

174

175

176

177

178

179

180

181

182

183

184

185

186

187

188

190

191

192

193

194

195

196

197

198

189

201

202

203

204

205

206

207

208

$$
E D\left(x_{1}, y_{1}, x_{2}, y\right)=\sqrt{\left(x_{1}-y_{1}\right)^{2}+\left(x_{2}-y_{2}\right)^{2}}
$$

The use of CF (a popular technique in RSs) to send personalised recommendations to users based on their behaviour has become widely used due to its superb role in recommending preferred items. The CF technique utilises product ratings provided by a collection of customers and recommends products that the target customer has not yet considered but will likely enjoy (Al-Hadi et al., 2020). The rating score (with a value between 1 and 5) is used to indicate if a person likes a product or otherwise. These values are arranged in a matrix as rows. Thereafter, the similarity values between the target customer and other customers in the matrix are calculated to predict the customer's interest in the products (Natarajan et al., 2020). In this work, the patients represent users and the generated output features of the MIRA platform represent items. The considered MIRA platform offers a vast range of features for several games and movements managed in a single matrix whereas, the preferences of customers are managed in the rating matrix for estimation via the $\mathrm{CF}$ technique. This matrix of features necessitates division using the $k$-means algorithm to minimise the prediction errors. Moreover, Algorithm 1 summarizes the procedure involved in the $\mathrm{CF}$ technique.

\section{Algorithm 1. Pseudocode of the CF technique}

Input:

Matrix features (patients, features)

Output:

A set of prediction scores for features

Steps:

- Choose the target patient from the matrix features.

- Assign the similarity between target patient and other patients utilising similarity distance.

- Assign the prediction value for each feature using the prediction approach.

- Measure the accuracy prediction performance using error function, such as Root Mean Squared Error (RMSE) (Al-hadi et al., 2020).

\section{b. k-means Algorithm}

The $k$-means is a clustering algorithm that is often used in iterative optimisation, given its efficiency (Zainal et al., 2019). Algorithm 2 displays the proximity measures of $k$-means which are city block, hamming, cosine, correlation coefficient, and squared Euclidean Distance (ED). The ED between two points is the length of the straight line that connects them. Within the Euclidean plane, the distance between points $\left(\mathrm{x}_{1}, \mathrm{y}_{1}\right)$ and $\left(\mathrm{x}_{2}, \mathrm{y}_{2}\right)$ can be calculated using Eq. (1) (Borja et al., 2018).
209

210

\section{a. Collaborative Filtering (CF)}




\section{c. K-Nearest Neighbours Algorithm}

a. Allocate each item d1 to the cluster with the nearest centroids (Eq. (1)).

b. For each cluster, calculate the new mean.

c. Calculate the junction among clusters to keep the $k$ number of clusters.

Until convergence criteria are met.

230

231

232

233

234

235

236

237

238

239

240

241

242

243

244

245

246

247

248

249

250

251

252

253

254

The K-NN is a simple classification method used to analyse a large matrix of features or to provide recommendations (Weisstein). When new data are required to be categorised, the K-NN algorithm computes the distance in values between the target record and other records. These records are ordered based on distance (Tarus, Niu \& Mustafa, 2018). At the final stage, the first $k$ record will be chosen from the ordered list i.e., K-NN. The pseudocode in Algorithm 3 describes the stages of applying K-NN.

\section{Algorithm 3. Pseudocode of K-nearest neighbours algorithm.}

Input:

Matrix Features (Patients, Features)

Output:

A set of neighbours most like the target patient.

Steps:

1- Choose the target patient from the matrix features.

2- Assign the similarity values amid the target patient and other patients by a similarity distance measure, as shown in Eq. (1).

3- Sort the patients starting from the lowest distance value to the topmost distance value (from the highest similarity to the lowest similarity).

4- Choose $k$ number of patients from the first in the sorted list.

\section{d. Bacterial Foraging Optimisation Algorithm}

Optimisation algorithms have proven effective in several areas including RS (Al-Hadi et al., 2017) and healthcare (Zainal et al., 2020). For instance, BFOA has been well-embraced in recent RS approaches for providing high accuracy prediction (Al-hadi et al., 2020)(Al-Hadi et al., 2017). 
255

256

257

258

259

260

261

262

263

264

265

266

267

268

269

270

$$
(j+1, k, l)=\beta^{i}(j, k, l)+C_{i}+\emptyset / \sqrt{\emptyset_{i}^{t} \emptyset_{i}}
$$

where $\beta^{i}$ represents the members of bacteria $i$ (i.e., latent features of patients), $C_{i}$ is the stage degree in the direction of the tumble, $j$ denotes the index for the sum of chemotactic, $k$ refers to the index for the number of reproductions, and $l$ reflects the index for the sum of elimination-dispersal. Besides, $\varnothing / \sqrt{\emptyset_{i}^{t} \emptyset_{i}}$ is the random unit length direction shown during the swimming phase. In the swarming mechanism, the latent features of a patient release attractant or repellent signals regarding other patient's latent features, as portrayed in Eq. (3).

$$
\begin{aligned}
& J_{c c}\left(\beta+\beta^{i}(j, k, l)\right) \\
& =\left[\sum_{i=1}^{S}-d_{\text {attract }} \exp \left(w_{\text {attract }} \sum_{m=1}^{P}\left(\beta_{m}-\beta_{m}^{i}\right)^{2}\right]+\left[\sum_{i=1}^{S}-h_{\text {repellant }} \exp \left(-w_{\text {repellant }} \sum_{m=1}^{P}\left(\beta_{m}-\beta_{m}^{i}\right)^{2}\right]\right.\right.
\end{aligned}
$$

271

272

273

274

275

276

277

278

279

280

281

282

283

where $d_{\text {attract }}$ is the depth of the attractant that can be recycled to establish the immensity of secretion of attractant by a cell value, $w_{\text {attract }}$ is the width of the attractant than can be recycled to denote the means by which the chemical cohesiveness of the signal diffuses, $h_{\text {repellant }}$ sets the height of the repellent (a propensity to avoid a nearby cell), and $w_{\text {repellant }}$ defines the negligible area where the cell is relative to the diffusion of the chemical signal. $S$ is the number of groups within the patients' latent features, $P$ denotes the dimension of the search space, $\beta_{\mathrm{m}}$ is the latent features of group number $m$, and $\beta_{m}^{i}$ represents latent feature number $i$ in group $m$.

b. Reproduction: This phase deals with the feedback (RMSE) value which acts as the fitness value. These values are obtained after training the target patients' features that have been extracted through the current training stage using the $k$-means, K-NN, BFOA and CF methods. The RMSE values will be saved in an array before sorting (smaller and larger values). The lower half of the latent features having a larger fitness value (dies) while the outstanding latent features or the other 
284 285

286

287

288

289

290

291

292

293

294

295

296

297

298

299

300

301

302

303

304

305

306

307

308

309

310

311

312

313

314

315

316

317

318

319

320

321

322

half of the population is separated into two equivalent parts having equal values. This phase keeps the bacteria population constant. Eq. (4) shows the healthy values for patients' latent features.

$$
J_{\text {health }}^{i}=\sum_{j=1}^{N_{c}+1} J(i, j, k, l),
$$

where $i$ is the sum of patients' latent features, $j$ is the sum of chemotactic steps $N_{c}$, $k$ is the reproduction step, and $l$ is the elimination-dispersal step.

c. Elimination-Dispersal: This phase provides the position shifting probability for the limited latent features of patients. The random vectors are produced and arranged in ascending order.

\section{Materials \& Methods}

In this study, ReComS, ReComS + and ReComS++ approaches are proposed to recommend preferences for the MIRA platform. These preferences are used to determine the input settings of the exergames by learning the precise behaviours of patients. ReComS integrates the K-NN and CF methods to classify the predicted values by reducing the error value. The error value is obtained using the projected and actual values of the previous session of the exergame. The ReComS+ approach improves the prediction performance of ReComS by integrating the $k$-means algorithm with K-NN and CF, which reduces the error value. However, this error value is relatively high. Hence, it lowers the prediction performance of the ReComS and ReComS +. ReComS ++ further reduces the error value by optimising the prediction or projection values and integrating $k$-means, $\mathrm{K}-\mathrm{NN}, \mathrm{CF}$, and BFOA algorithms.

\section{a. Dataset}

This study was carried out in the rehabilitation centre of Melaka, Malaysia to analyse the generated data using MIRA platform with ethic approval no PRPTAR.600-5(27) by Pusat Rehabilitasi Perkeso Sdn. Bhd. The MIRA platform patient data file in this study contains patients' personal information such as first and last names, patient ID, and birth date. It also entails information related to the games played such as the session ID, name of the game, movement ID, movement name, and associated dates (Wilson et al., 2017). Each selected game and movement acts as one exergame with its unique input variables in the item settings dialogue. The settings include the sides used (left or right), duration, difficulty, tolerance, minimum and maximum ranges. The values of these variables could be fixed based on the default values or adjusted by the physiotherapist after evaluating the performance of the patient. The MIRA platform could generate 26 variables based on the exergame or cognigame (a game that trains the cognitive function). Table 1 describes the most significant variables generated by the exergames.

The experimental data contained 3553 records generated by 61 patients with different types of diagnoses in which 41 patients had a stroke, 14 patients had TBI, seven patients had SCI, one patient had CP, and two patients had humerus. Patients provided written informed consent before the start of each experiment. Fig. 2 portrays an example of the MIRA setting for animals' exergame with the elbow flexion movement. The item settings includes six variables that can be manipulated 
323

324

325

326

327

328

329

330

331

332

333

334

335

336

337

338

339

340

341

342

343

344

345

346

347

348

349

350

351

352

353

354

355

356

357

358

359

360

361

362

363

by the physiotherapist or player. Table 2 presents a description of the generated exergame features by patients using the MIRA platform. In each session, a patient plays an exergame by moving his/her limbs according to the rules of the game and movement exercise. During the exercise, the physiotherapist predicts the variables of the input setting such as difficulty, tolerance, minimum range, and maximum range according to his observation or adopts the default values, but a number of patients experienced difficulty playing the games. Afterwards, he deduced the accurate settings from the previous performance of patients in each exergame. As compared to using the default settings, a more accurate setting ensures patients play better. This indicates the significance of this research to the MIRA platform.

In this study, the ReComS approach is proposed to predict the variables of the input setting according to the data history of the patient. As ReComS is expected to provide low prediction accuracy, it is integrated with a clustering method (as used in similar experimental works (AlHadi et al., 2017) (Al-Hadi et al., 2020)) and referred to as the ReComS+ approach. ReComS+ provides good prediction accuracy. ReComS ++ is developed by further integrating $\mathrm{ReComS}+$ with the BFOA algorithm to learn the latent features of the patients and to lower the RMSE value throughout the learning iteration process. The experimental results of ReComS and ReComS+ are utilised to benchmark the prediction performance of the ReComS++ approach.

\section{b. ReComS Approach}

Most personal recommendation systems use the $\mathrm{CF}$ and the $\mathrm{K}-\mathrm{NN}$ for providing personal recommendations. Here, the $\mathrm{CF}$ technique provides the target patient with personal recommendations according to the common behaviours of other patients. K-NN method is used to obtain the nearest neighbours of each target patient based on their similarities (Portugal, Alencar \& Cowan, 2018). Thus, ReComS integrates the $\mathrm{K}-\mathrm{NN}$ algorithm with the CF technique for learning the personal behaviour of patients and predicting the input setting variables. The proposed ReComS approach assists the physiotherapist to collect accurate data from patients who need to play exergames using MIRA platform.

The framework of ReComS is arranged following the steps in Fig. 3. The ReComS approach is set to the target patient to manage the entire patients' features in the features matrix. $\mathrm{K}-\mathrm{NN}$ is applied to classify the $k$ nearest neighbours based on the similarities between the target patient and other patients. The RMSE function calculates ReComS prediction accuracy based on the distance between the features of the target patient and prediction values obtained using this approach.

\section{d. ReComS+ Approach}

$\mathrm{ReComS}+$ is proposed to improve the prediction accuracy of ReComS. The ReComS approach should yield higher accuracy, even if the RMSE remains high. $k$-means, $\mathrm{K}-\mathrm{NN}$, and CF methods are integrated into ReComS + to provide the predicted variables as input setting in the MIRA dialogue box of each exergame. The $k$-means algorithm clustered the records of patients into the sum of clusters $(k)$. The cluster that contained the record of the target patient is selected to obtain the matrix of neighbours that are integrated with the CF to provide the preferences values, as shown in Fig. 4.

\section{e. ReComS++ Approach}


Despite the high accuracy of $\mathrm{ReComS}+$ prediction, its error value is relatively high. This error value can be reduced by deploying learning methods. Thus, BFOA is integrated with the $\mathrm{ReComS}+$ for learning the behaviours of neighbours by lowering the error value during the iteration stages. The framework of ReComS++ encapsulates four stages that are needed to provide the preferences for patients' input settings, as shown in Fig. 5. These stages are described as follows:

\section{i. Data preparation}

- $\quad$ Reading data records and arranging variables in a matrix.

- Encoding the textual data (such as gender, diagnosis, game name, difficulty and side) using numbers.

- $\quad$ Normalising the generated variables of each exergame based on the duration by applying Eq. (5).

$F=F * T_{d} / T_{F}$

- Normalising the variables into the range between 0 and 1. This is based on the dimension of each variable, features rescaling and the need to provide proper compatible values for machine learning algorithms. The normalization is performed based on the standard data mining requirement to provide accurate variables approximation and prediction using Eq. (6).

$$
F_{i j}=\frac{F_{i j}-X}{\mathrm{Y}-\mathrm{X}}(\partial-\varnothing)+\emptyset
$$

where $F_{i j}$ is the value of record $i$ and variable $j, X$ is the least value, and $Y$ is the highest value in the whole matrix of variables. $\partial$ is the highest target value (1) and $\emptyset$ is the least target value (0).

- Assigning the target patient, target movement, and side, to find the latest record of the patient where he played the selected movement exergame on the target side. Variables of this record would be arranged in the first row in the matrix of features.

- Selecting whole records from data containing the target movement of the target patient and putting the variables of these records in the matrix of features.

- The matrix of features would be divided into two parts. The first part would have $70 \%$ of records for the purpose of training and the second part would have $30 \%$ for evaluations.

\section{ii. Clustering by $k$-means algorithm}

The MIRA data consists of 30 games and 34 movements that provide 1020 kinds of features, which make it challenging to analyse. The features are grouped into a single matrix. Hence, the $k$-means 
399

400

401

402

403

404

405

406

407

408

409

410

411

412

413

414

415

416

417

418

419

420

421

422

423

424

425

426

427

428

429

430

431

432

433

434

435

436

437

438

439

440

clustering algorithm is used to simplify the various types of features generated by playing the games and movements in the MIRA application. These features are divided into a set of clusters. The challenge of this experimental work is in determining the accurate sum of clusters. To address this issue, MIRA data are tested in a set of $k$ clusters ranging between 5 and 10 . Based on the data collected, we assign the range from 5 to 10 clusters as the sufficient range of clusters. This is intended to avoid the $k$-means clustering problem ( $k$ problem) when using clusters above 10 because of the high number of zeros in each matrix of features. After that, the prediction performed by ReComS is assessed based on this set of clusters.

\section{iii. Classification by K-NN algorithm}

The K-NN is applied to retrieve similar records to the target record in the matrix. The challenge in this process is in determining the accurate sum of neighbours. A small number of neighbours yields an accurate prediction performance while a vast number of neighbours exhibits the lowest performance. However, the few numbers of neighbours are not sufficient to learn the accurate features of patients. Thus, a larger number is required to accurately learn the patients' features. Addressing this problem, BFOA is integrated with the ReComS + to improve the prediction accuracy. In this experimental work, the K-NN algorithm applies the squared ED, as given in Eq. (7).

$$
D(x-y)=\sqrt{\sum_{i=1}^{n}(x i-y i)^{\wedge} 2},
$$

where $D$ refers to the distance value between records $x$ and $y$ while $n$ denotes the number of features. Eq. (7) calculates the distance between the target record and the total records of the target cluster. The target record is hinged on the target cluster mainly because the distance between the target record and the centroid point of this cluster is smaller when compared to other clusters. After calculating the distance between the total records of the target cluster and the target record, the values are sorted in ascending order to derive closely related neighbours to the target record. Records having small values of distance have the highest similarity value to the target record thus, being the nearest neighbours. In this experimental work, the $k$ of neighbours is determined based on a set of $k$, i.e., 25 neighbours, 50 neighbours, 75 neighbours and 100 neighbours. These four numbers of neighbours have been chosen according to the available features of each exergame within each cluster. These features constitute an effective solution for executing the required training processes for three reasons. First, most patients like to play only a few interesting MIRA exergames. Thus, other exergames have fewer records. Meanwhile, the machine learning algorithms need a large number of records to facilitate the process of learning the latent-features using the exergame output features. Second, interesting exergames can be predicted easily due to their rich output clusters using most similar features to the target exergame. Then, K-NN method can find $k$ neighbours of over 100 records while it is more difficult in clusters with less than or equal 100 records. Third, some patients need to play specific exergames to improve their idle movement skills. The number of output records of these specific exergames is small. Thus, the output cluster of the target matrix of such special exergame does not converge due to the gap between them and the popular exergames. Hence, there is only a few neighbours such as 25 or 50 from these output clusters while it is quite difficult to find 75 or 100 neighbours. In addition, more than 100 neighbours can be considered as impossible or inaccurate due to the resulting poor convergence between the features of target exergame and those of poor-performing clusters. Each 
441

442

443

444

445

446

447

448

449

450

451

452

453

454

455

456

457

458

459

460

461

462

463

464

465

466

467

468

469

470

471

472

473

474

475

476

477

478

479

$k$ neighbour is then evaluated using ReComS based on its prediction performance in comparison to other numbers of neighbours.

\section{f. Developing CF Performance with BFOA}

The notion of predicting values for variables that constitute the item settings in MIRA, based on the behaviour of patients that have played a few exergames, is similar to the idea of predicting products for customers based on their preferences in the recommendation system that uses the CF technique. Obviously, the CF predicts the score values for products while ReComS predicts variables for all input and output features associated with a specific game and a peculiar movement. Typically, the CF technique uses three functions to estimate values, as described next.

\section{- Similarity Function}

This function provides the correlation between the target record (of game and movement) and total records. The similarity functions that apply the CF technique are Cosine Similarity (Al-hadi et al., 2020) and the Pearson Correlation Coefficient (Srifi et al., 2020). Note that when using the Cosine or Correlation coefficient for MIRA data, these functions generate some outliers due to the existence of zeros in the feature matrix. Thus, the Euclidean similarity function, as shown in Eq. (7), emerges as the most suitable similarity function to be applied for MIRA data, mainly because all the calculated similarity values are known.

\section{- Prediction Function}

This is an important computational procedure obtained from the similarity values retrieved from the similarity function and the correlation between the total records. For the purpose of prediction in this experimental work, Eq. (8) has been proposed based on the current prediction function in the CF technique (Natarajan et al., 2020) after considering the difference between rating scores and features values of MIRA.

$$
P_{i}=V_{a}+\frac{\sum_{h=1}^{N} D\left(F_{a}, F_{h}\right)\left(F_{h, i}-V_{h}\right)}{\sqrt{\sum_{h=1}^{N} D\left(F_{a}, F_{h}\right)}},
$$

where $P_{i}$ is the predicted or projected value for feature $i, V_{a}$ is the average value of all feature values for the target record, $N$ is the sum of neighbours, $D$ is the distance similarity value between $F_{a}$ (feature value of target record) and $F_{h}$ (feature value of neighbour $h$ ). Also, $F_{h, i}$ refers to the feature value $i$ of the neighbour $h$, whereas $V_{h}$ denotes the average value of all features of neighbour $h$.

In this work, Eq. (8) is used in ReComS and ReComS+ by employing the error function. Nevertheless, the generated output still has errors and the predicted values are over-fitted. Such over-fitting occurs when the predicted value is larger than the features generated by the target exergames. Fig. 6 graphically exemplifies the generated features of the target exergame and the features predicted by the $\mathrm{CF}$ technique within the procedures of the ReComS approach.

In Fig. 6, eight predicted feature values are overfitted because these values are greater than the feature values of the target exergames. The remaining predicted features have lower fitting values due to their values are smaller than the feature values of the target exergames. Hence, the predicted features need to be normalised to fit/align these values with the target exergame features. 
480

481

482

483

484

485

486

487

488

489

490

491

492

493

494

495

496

497

498

499

500

501

502

503

504

505

506

507

508

509

510

511

512

513

514

515

516

Nonetheless, the ReComS and ReComS+ are inaccurate approaches in normalizing the predicted features. Thus, an optimised algorithm is embedded in the prediction method to normalise the prediction values. Notably, BFOA has been acknowledged as an optimisation algorithm commonly applied in recommendation systems (Hwangbo, Kim \& Cha, 2018) since this algorithm can exceptionally learn the deep features of each matrix. The contribution of the ReComS ++ approach is represented in Eq. (9).

$$
P_{i}=V_{a}+\frac{B_{i} \sum_{h=1}^{N} D\left(F_{a}, F_{h}\right)\left(F_{h, i}-V_{h}\right)}{\sqrt{\sum_{h=1}^{N} D\left(F_{a}, F_{h}\right)}},
$$

- where $B_{i}$ is the bacteria value that can be learned by tracking feature $F_{i}$, and the sum of bacterium members will be equal to the number of neighbours' features. These bacteria have been used to track all features of the neighbours (such that each column in the matrix is managed by a bacterium member) and provide accurately predicted input variables for MIRA. The remaining vectors of Eq. (5) have been described in Eq. (4). The BFOA is implemented based on the algorithmic phases described in Section 2.5 while the values of bacteria factors are listed in Table 3 .

\section{- Benchmark Function}

The proposed approaches are evaluated to examine the performance of the CF technique using RMSE (Al-Hadi et al., 2020) and Mean Absolute Error (MAE) (Wang, Yih \& Ventresca, 2020). In this article, RMSE measure is used for calculating the differences between the variable of target patient and predicted values for same variables of target patient. These variables are the input setting variables (difficulty, tolerance, minimum range, and maximum range) and the generated variables by exergame such as average acceleration, average deceleration, moving time and other variables that are described in Table 1. The predicted values are calculated by Equation 9 according to the similarity values for patients comparing to the target patient variables.

In other words, the total number of generated exergame features of all patients are divided into $k$ clusters using the $k$-means. Each cluster is classified by the K-NN algorithm that selects the most suitable features needed to provide accurate feedback. BFOA focuses on accurately learning the latent features. This is achieved by tracking the positive effects produced through classified features by reducing the RMSE value throughout the optimization stages. This is achieved by accurately learning the convergence among the generated features for various patients who played various exergames, as shown in Eq. (10).

$$
R M S E=\frac{1}{R} \sum_{p=1}^{R} \sqrt{\frac{1}{n} \sum_{i=1}^{n}\left(F_{a, i}-P_{i}\right),}
$$

where $R M S E$ is the average $R M S E$ values for all records in the matrix of neighbours, $R$ denotes the number of records in the training or testing sets, $n$ refers to the number of features in the matrix, $F_{a, i}$ represents the value of feature $i$ in the target record, and $P_{i}$ stands for the predicted value recommended to feature $F_{i}$. The highest RMSE value reflects the lowest accuracy prediction performance.

\section{Results}

ReComS improves the performances of the following three experimental procedures.

\section{a. Evaluating the ReComS Approach}


517 The K-NN algorithm classifies integral features of a vast number of patients who played several 518 games and with varying movements. The CF computes the similarity between the target features 519 and the features of each neighbour before predicting the new values. The performance of the predicted values is consequently assessed using the RMSE value. To benchmark the varied RMSE values for several target records based on patients' personality behaviours, the average RMSE is calculated to obtain accurate outputs. Fig. 7 illustrates the prediction performance accuracy using this approach based on two sets, training and testing sets. Both sets show that 25 of the nearest neighbours yields the lowest performance accuracy when compared to those having 50, 75, and 100 neighbours. The case of 100 neighbours yielded more accurate performance when compared to that of 25 neighbours. This deteriorates the prediction performance of the nearest neighbours, which may be solved by other classification approaches.

\section{b. Evaluating the ReComS+ Approach}

529

530

531

532

533

534

535

536

537

538

539

540

541

542

543

544

545

546

547

548

549

550

551

552

553

554

555

556

557

558

559

The $k$-means clustering algorithm is applied in ReComS + to address the limitation of ReComS using CF and K-NN. Deploying these methods, even the use of a small number of nearest neighbours could result in a highly accurate performance. Fig. 8 indicates that after integrating the $k$-means algorithm into ReComS, the prediction performance of CF is improved and the feedback of the nearest neighbours are corrected. The outcomes, as depicted in Fig. 8, show the prediction performance of CF using five clusters and 25 neighbours is better for both the training and testing sets, as compared to the results when 50, 75, and 100 neighbours are integrated with five clusters. Nevertheless, the number of clusters is not justified, as six or ten clusters may offer a higher prediction accuracy as shown in Fig. 9. Hence, ReComS + tests the prediction performance using $k$ clusters to address the problem associated with cluster numbers.

In addition, Fig. 9 proves that various numbers of clusters can provide similar prediction performance as ReComS + for both the training and testing sets. The results show that 5 clusters provide the highest accuracy prediction using the training dataset when compared to the performance of ReComS + by the other $k$ clusters, which ranged between 6 and 10. The accurate prediction of $\mathrm{ReComS}+$ in the testing dataset, therefore, confirms that the prediction performance of $\mathrm{ReComS}+$ for all $k$ clusters is similar to that of 6 clusters that generate low accuracy performance. For this reason, subsequent experimental works using ReComS ++ apply 5 clusters. Though the accuracy performance of ReComS + has been improved using this approach, the RMSE is still high and the range of the predicted values should be normalised. For this reason, the BFOA is applied to normalise the predicted values and minimise RMSE values.

\section{c. Evaluating ReComS++ Approach}

The BFOA is implemented in this work to normalise the predicted values of the ReComS+ approach, which utilised the CF, K-NN and $k$-means methods. The results proved that the predicted values are similar to the variables of the target record while emphasizing the need to decrease RMSE values. Fig. 10 presents the prediction performance of the ReComS++ approach that employed CF, K-NN, $k$-means, and BFOA for both training and testing sets. The RMSE values, through this approach, appeared to be small, thus indicating high prediction accuracy for all sets of tested neighbours. The set of 25 neighbours provide the highest prediction accuracy when compared to the other sets of neighbours (i.e., 50, 70 and 100) for the MIRA training and testing datasets. The results of both training and testing sets are close, indicating that this approach provides accurate prediction values for the whole target records in the MIRA data. 
560

561

562

563

564

565

566

567

568

569

570

571

572

573

574

575

576

577

578

579

580

581

582

583

584

585

586

587

588

589

590

591

592

593

594

595

596

597

598

599

600

601

602

603

\section{Discussion and Future works}

The CF technique is applied to predict the values of future variables of the item settings. This technique is integrated with the K-NN algorithm in ReComS. It provides each exergame with predicted feature values related to the generated features of the target exergame. Few of these predicted features can be used to assign the variables of the exergame dialogue box setting. The ReComS approach provides a low prediction accuracy due to the high percentage of the overfitted predicted values. Hence, ReComS needs further improvement to classify the various output features of all exergames. For this reason, ReComS is subsequently improved by ReComS + that utilizes the $k$-means algorithm for grouping the various generated features into $k$ clusters and then finding the nearest features to the target exergame features within the same cluster.

ReComS + approach chooses the best cluster and number of neighbours through its accurate predictions. The prediction performance of ReComS + is more accurate than that of ReComS because the former accurately learn the convergence among exergame features using the $k$-means. Similarly, each cluster is classified to accurately learn the neighbours' features using K-NN. Nevertheless, the predicted values generated in ReComS + vary and the prediction accuracy of $\mathrm{ReComS}+$ is low due to the difficulty in learning the latent features of the vast amount of generated exergame features. Thus, the ReComS + approach should be further improved using an optimization algorithm that can effectively learn the latent features of the neighbours within each cluster.

BFOA is one of the efficient optimisation algorithms that have been used in improving the prediction performance of the CF technique in some recommendation systems (Al-hadi et al., 2020)(Al-Hadi et al., 2017). Accordingly, BFOA is utilized in the ReComS++ approach for reducing the overfitted prediction values by learning the latent features of the neighbours within each cluster. Further, BFOA is used to ensure the outlier data belonging to the cluster. The experimental approaches show that the ReComS++ approach has addressed the inherent challenges of the first and second approaches. Fig. 11 shows the comparisons between the RMSE values of the three experimental approaches: ReComS, ReComS+, and ReComS ++ by MIRA training sat. The ReComS++ approach provides the lowest RMSE value, indicating it has highest prediction accuracy when compared with both ReComS and ReComS + approaches. Furthermore, Fig. 11 illustrates the experimental outcomes derived from the MIRA testing dataset. The results are similar to those generated for the training dataset using the ReComS++ approach. This indicates that ReComS ++ successfully addressed the drawbacks of the ReComS and ReComS+ approaches.

There are several potential promising directions where ReComS ++ approach can be integrated with other platforms that have the profile settings for exergames. It may be used to learn latent features of the exergames output features by each platform that has settings variable for predicting the input settings' variables. Further, ReComS ++ approach provides $85 \%$ of prediction results is correct while this result can be upgraded up to $90 \%$ by exploring other machine learning methods to reduce the computational time of $\mathrm{ReComS}++$ approach throughout the iteration learning. It can focus on specific variables such as average correct answer reaction time for cognitive. The cognitive and the repetition of range of motion need more study to understand the suddenly patient movements and predicting the suitable settings. Therefore, we trend to explore various linear regression techniques for predicting the optimal setting variables. In addition, we trend to explore several structures of deep learning methods to find the best structure that can be reducing the 
604 computational time and learning more accurate latent features of patients based on their personal 605 behaviours during playing the exergames.

\section{6 a. Criteria for the ReComS++ approach according to the output}

607

608

609

610

611

612

613

614

615

616

617

618

619

620

621

622

623

624

625

626

627

628

629

630

631

632

633

634

635

636

637

638

639

640

641

642

643

644

645

646

A significant milestone in this work is determining the predicted difficulty level and the remaining variables in the dialogue box for the setting of each item. Fig. 12 shows the minimum and maximum predicted values for the difficulty, tolerance, as well as minimum and maximum ranges. Based on the predicted and observed values obtained from the MIRA application in Melaka, Malaysia while supervising patients who played MIRA games. The determined threshold intervals are as illustrated in Fig. 13 where this figure presents two observations for two target records created by the output of the predicted variables using ReComS++. First, the example in Fig. 13 depicts the actual observation made by the physiotherapist for a patient who has played the game with movement exercise at an easy (difficulty) level with a tolerance up to $30 \%$ (percentage of range of movement is $0-60 \%$ ). After that, the experimental approach normalises these values into the range between 0 and 1, as shown in the figure. The experimental approach performs closely to the actual values in terms of difficulty, tolerance, minimum range, and maximum range. Based on the threshold shown in Fig. 12, the experimental approach determines the final prediction values of the next item settings as Easy; 20 for tolerance, 0 for min range and $70 \%$ for max range. The second example depicts similar procedures needed to arrive at the final decision in accordance with the threshold.

\section{b. Evaluating the $\mathrm{ReComS}++$ approach based on the physiotherapist observations}

The ReComS++ approach, programmed using Java, is included in the MIRA system to provide physiotherapists and patients with preferences. Fig. 14 shows the interface of the preferences where the physiotherapist (who helps patients to play MIRA exergames) could easily obtain the recommended preferences for the selected patient, movement, and side. On evaluating the ReComS++ approach, we obtain the evaluation file completed by physiotherapists of Perkeso Rehabilitation Centre in Melaka, Malaysia. The file records the physiotherapists' observations after using the preferences suggested by ReComS++ for a set of patients over a period of 5 weeks. The file contains 1182 records. Table 4 reveals an example of the information provided in the file.

Table 4 presents the set of movements performed by a group of patients who play a set of exergames (movements and games) using the MIRA platform. Each exergame has four preferences that constitute the input setting variables in ReComS++ (i.e., difficulty, tolerance, minimum range and maximum range). Here, the physiotherapist observes each patient who performs the exergame and registers his/her activity performance as positive $(\mathrm{P})$ or Negative $(\mathrm{N})$. The evaluation results are summarised in Table 5. The table shows a higher percentage of positive preferences compared to negative preferences. This implies that ReComS effectively recommends accurate preferences for patients.

\section{Conclusion}

In most cases, patients train their disabled limbs by utilising the facilities offered at rehabilitation centres to regain their limbs functionality. The VRT, such as MIRA, refers to a contemporary rehabilitation technique that aids patients to perform "game-aided" exercises in order to increase their motivation and engagement in physical therapy. Nonetheless, physiotherapists who deal with this application need to predict the values of the input variables of the item settings for each patient 
647

648

649

650

651

652

653

654

655

656

657

658

659

660

661

662

663

664

665

666

667

668

669

670

671

672

673

674

675

676

677

678

679

680

681

682

683

684

685

686

687

688

689

690

691

692

manually, which is the main challenge in this domain. Therefore, in this study, we utilise a recommender system to suggest the most suitable settings for patients' movements based on their movement history. Since the exergames generate various features, automated analysis is required to provide a summary of the patient's (movement) performance. To address these challenges, three experimental approaches: 1) ReComS with the CF and K-NN approach; 2) ReComS+ with the CF, $\mathrm{K}-\mathrm{NN}$; and 3 ) $k$-means approach, in addition to $\mathrm{ReComS}++$ with the $\mathrm{CF}, \mathrm{K}-\mathrm{NN}, k$-means and the BFOA approach; were proposed and their shortcomings were tested by learning procedures. The experimental results demonstrated that ReComS + yields more accurate predictions when compared with ReComS while ReComS++ achieves a higher accuracy as compared to ReComS + . Overall, ReComS++ performs best for MIRA exergames as it provides MIRA with the most accurate predictions for the input setting dialogue box. It thus assists patients to perform MIRA exergames correctly.

\section{Acknowledgements}

The authors wish to appreciate the editor and anonymous referees for their constructive comments and criticism. This work is supported by the Newton-Ungku Omar Fund from Malaysia Industry-Government Group from High Technology (MIGHT) and Grant code USIM/INTNEWTON/FST/IHRAM/053000/41616.

\section{References}

Al-Hadi IAA-Q, Ahmed M, M. E, Ali W, M S. 2020. Temporal-based Optimization to Solve Data Sparsity in Collaborative Filtering. International Journal of Advanced Computer Science and Applications 12(11):514-522. DOI: 10.14569/ijacsa.2020.0111262.

Al-hadi IAA-Q, Sharef NM, Mustapha N, Nilashi M. 2020. Latent based temporal optimization approach for improving the performance of collaborative filtering. PeerJ Computer Science:1-25. DOI: $10.7717 /$ peerj-cs.331.

Al-Hadi IAA-Q, Sharef NM, Sulaiman MN, Mustapha N. 2017. Bacterial foraging optimization algorithm with temporal features to solve data sparsity in recommendation system. Proceedings of the Second International Conference on Internet of things, Data and Cloud Computing (ACM) 148:1-6.

Amghar YT, Fizazi H. 2017. A Hybrid Bacterial Foraging Optimization Algorithm and a Radial Basic Function Network for Image Classification. Journal of Information Processing Systems. 13(2):215-235.

Baur K, Schättin A, de Bruin ED, Riener R, Duarte JE, Wolf P. 2018. Trends in robot-assisted and virtual reality-assisted neuromuscular therapy: a systematic review of health-related multiplayer games. Journal of neuroengineering and rehabilitation. 15(1):1-19. DOI: 10.1186/s12984-018-0449-9.

Borja EF, Lara DA, Quevedo WX, Andaluz VH. 2018. Haptic Stimulation Glove for Fine Motor Rehabilitation in Virtual Reality Environments. In International Conference on Augmented Reality, Virtual Reality and Computer Graphics. Springer, Cham.211-229. DOI: 10.1007/978-3-319-95282-6_16.

Brokaw EB, Brewer BR. 2013. Development of the home arm movement stroke training environment for rehabilitation (HAMSTER) and evaluation by clinicians. International Conference on Virtual, Augmented and Mixed Reality. Springer, Berlin, Heidelberg. 22-31. DOI: 10.1007/978-3-642-39420-1-3.

Chi B, Chau B, Yeo E, Ta P. 2019. Virtual reality for spinal cord injury-associated neuropathic pain: Systematic review. Annals of Physical and Rehabilitation Medicine. 62(1):49-57. 
693

694

695

696

697

698

699

700

701

702

703

704

705

706

707

708

709

710

711

712

713

714

715

716

717

718

719

720

721

722

723

724

725

726

727

728

729

730

731

732

733

734

735

736

737

738

DOI: 10.1016/j.rehab.2018.09.006.

Covarrubias M, Bordegoni M, Rosini M, Guanziroli E, Cugini U, Molteni F. 2015. VR system for rehabilitation based on hand gestural and olfactory interaction. In Proceedings of the 21st ACM Symposium on Virtual Reality Software and Technology.117-120. DOI: $10.1145 / 2821592.2821619$.

Czakó N, Silaghi C, Vizitiu RS. 2017. Rehabilitation of a 7-year-old Boy with Brachial Plexus Palsy Using MIRA Exergames and Vojta Therapy. E-Health and Bioengineering Conference (EHB).349-352.

Díaz I, Gil JJ, Sánchez E. 2011. Lower-Limb Robotic Rehabilitation: Literature Review and Challenges. Journal of Robotics. 2011:1-11. DOI: 10.1155/2011/759764.

Da Gama AEF, Chaves TM, Figueiredo LS, Baltar A, Meng M, Navab N, Teichrieb V, Fallavollita P. 2016. MirrARbilitation: A clinically-related gesture recognition interactive tool for an AR rehabilitation system. Computer Methods and Programs in Biomedicine. 135:105-114. DOI: 10.1016/j.cmpb.2016.07.014.

Hwangbo H, Kim YS, Cha KJ. 2018. Recommendation system development for fashion retail ecommerce. Electronic Commerce Research and Applications. 28:94-101. DOI: 10.1016/j.elerap.2018.01.012.

Ismail W, Zamin N, Hanafi MH, Mohamad AH. 2019. A Conceptual Model of Hybrid Monitoring Rehabilitation Progress of Stroke Patients: A Case Study of a Public Tertiary Hospital in Malaysia. IEEE 6th International Conference on Industrial Engineering and Applications (ICIEA), Tokyo, Japan.516-520. DOI: 10.1109/IEA.2019.8714999.

Jaarsma T, Klompstra L, Ben Gal T, Ben Avraham B, Boyne J, Bäck M, Chialà O, Dickstein K, Evangelista L, Hagenow A, Hoes AW, Hägglund E, Piepoli MF, Vellone E, Zuithoff NPA, Mårtensson J, Strömberg A. 2020. Effects of exergaming on exercise capacity in patients with heart failure: results of an international multicentre randomized controlled trial. European Journal of Heart Failure.1-11. DOI: 10.1002/ejhf.1754.

Li J, Erdt M, Chen L, Cao Y, Lee SQ, Theng YL. 2018a. The Social Effects of Exergames on Older Adults: Systematic Review and Metric Analysis. Journal of medical Internet research. 20:1-12. DOI: 10.2196/10486.

Li J, Erdt M, Chen L, Cao Y, Lee SQ, Theng YL. 2018b. The Social Effects of Exergames on Older Adults: Systematic Review and Metric Analysis. Journal of medical Internet research. 20(6):1-12. DOI: 10.2196/10486.

Maciejasz P, Eschweiler J, Gerlach-Hahn K, Jansen-Troy A, Leonhardt S. 2014. A survey on robotic devices for upper limb rehabilitation. Journal of NeuroEngineering and Rehabilitation. 11:1-29. DOI: 10.1186/1743-0003-11-3.

Mcglinchey M, Curtis A, Ritchie R, Jones GD. 2015. Feasibility of using MIRA with adult patients presenting with upper limb motor dysfunction post neurological damage. Proceedings of the 13th European Conference on the Advancement of Assistive Technology (AAATE).696-702. DOI: 10.3233/978-1-61499-566-1-696.

Merilampi S, Koivisto A, Sirkka A, Raumonen P, Virkki J, Xiao X, Min Y, Ye L, Chujun X, Chen J. 2017. The cognitive mobile games for older adults-A Chinese user experience study. In 2017 IEEE 5th International Conference on Serious Games and Applications for Health (SeGAH). IEEE. 1-6. DOI: 10.1109/SeGAH.2017.7939280.

Moldovan IM, Tric L, Ursu R, Podar A, Călin AD, Cantea AC, Dascălu LA, Mihaiu CA. 2017. Virtual rehabilitation programme using the MIRA platform, Kinect and Leap Motion sensors in an 81 years old patient with ischemic stroke. In $2017 \mathrm{E}-$ Health and Bioengineering

Peer) Comput. Sci. reviewing PDF | (CS-2020:10:53958:2:0:NEW 11 May 2021) 
Conference (EHB). IEEE. 325-328. DOI: 10.1109/EHB.2017.7995427.

Natarajan S, Vairavasundaram S, Natarajan S, Gandomi AH. 2020. Resolving data sparsity and cold start problem in collaborative filtering recommender system using Linked Open Data. Expert Systems with Applications. 149:1-9. DOI: 10.1016/j.eswa.2020.113248.

Naveen S, Sathish Kumar K, Rajalakshmi K. 2015. Distribution system reconfiguration for loss minimization using modified bacterial foraging optimization algorithm. International Journal of Electrical Power and Energy Systems. 69:90-97. DOI: 10.1016/j.ijepes.2014.12.090.

Portugal I, Alencar P, Cowan D. 2018. The use of machine learning algorithms in recommender systems: A systematic review. Expert Systems with Applications. 97:205-227. DOI: 10.1016/j.eswa.2017.12.020.

Pruna E, Corrales G, Catherine G, Mena L. 2018. Proposal for Muscle Rehabilitation of Lower Limbs Using an Interactive Virtual System Controlled Through Gestures. In International Conference on Augmented Reality, Virtual Reality and Computer Graphics. Springer, Cham.60-77. DOI: 10.1007/978-3-319-95282-6.

Radman A, Ismail W, Bahari M. 2018. Robotic devices for upper limb stroke rehabilitation: Potential research trends. In 2017 IEEE International Symposium on Robotics and Intelligent Sensors (IRIS). IEEE. 383-388. DOI: 10.1109/IRIS.2017.8250153.

Srifi M, Oussous A, Lahcen AA, Mouline S. 2020. Recommender systems based on collaborative filtering using review texts-A survey. Information. 11(6):1-21. DOI: 10.3390/INFO11060317.

Tarus JK, Niu Z, Mustafa G. 2018. Knowledge-based recommendation: a review of ontologybased recommender systems for e-learning. Artificial Intelligence Review. 50(1):21-48. DOI: $10.1007 / \mathrm{s} 10462-017-9539-5$.

Tousignant M, Giguère A, Morin M, Pelletier J, Sheehy A, Cabana F. 2014. In-home telerehabilitation for proximal humerus fractures: a pilot study. 6:31-38.

Turolla A, Dam M, Ventura L, Tonin P, Agostini M, Zucconi C, Kiper P, Cagnin A, Piron L. 2013. Virtual reality for the rehabilitation of the upper limb motor function after stroke: A prospective controlled trial. Journal of NeuroEngineering and Rehabilitation. 1(10):1-9. DOI: $10.1186 / 1743-0003-10-85$.

Wang D, Yih Y, Ventresca M. 2020. Improving neighbor-based collaborative filtering by using a hybrid similarity measurement. Expert Systems with Applications. 160:1-24. DOI: 10.1016/j.eswa.2020.113651.

Weisstein W. Distance. From MathWorld--A Wolfram Web Resource. Retreived from :http://mathworld.wolfram.com/Distance.html. (3rd April 2011).

Wilson JD, Khan-Perez J, Marley D, Buttress S, Walton M, Li B, Roy B. 2017. Can shoulder range of movement be measured accurately using the Microsoft Kinect sensor plus Medical Interactive Recovery Assistant (MIRA) software?. Journal of Shoulder and Elbow Surgery. 26(12):1-8. DOI: 10.1016/j.jse.2017.06.004.

$\mathrm{Xu} \mathrm{K}$, Zhang W, Yan Z. 2018. A privacy-preserving mobile application recommender system based on trust evaluation. Journal of Computational Science. 26:87-107. DOI: 10.1016/j.jocs.2018.04.001.

Yang C, Ji J, Liu J, Yin B. 2016. Bacterial foraging optimization using novel chemotaxis and conjugation strategies. Information Sciences. 363:72-95. DOI: 10.1016/j.ins.2016.04.046.

Zainal N, Al-Hadi IAAQ, Ghaleb SM, Hussain H, Ismail W, Aldailamy AY. 2020. Predicting MIRA Patients' Performance Using Virtual Rehabilitation Programme by Decision Tree 
785 Modelling. In: Recent advances in intelligent systems and smart applications. Cham:

786 Springer. 451-462. DOI: 10.1007/978-3-030-47411-9_24.

787 Zainal N, Faeid MZM, Kahaki SMM, Hussain H, Bahari M, Ismail W. 2019. Prediction scoring

788 in exergames for rehabilitation patients using K-means clustering. In 2019 6th International

789 Conference on Research and Innovation in Information Systems (ICRIIS), IEEE. 1-6. DOI:

$790 \quad 10.1109 /$ ICRIIS48246.2019.9073659.

791 
Figure 1

The case of a patient playing three game-based exercises using the MIRA Platform.

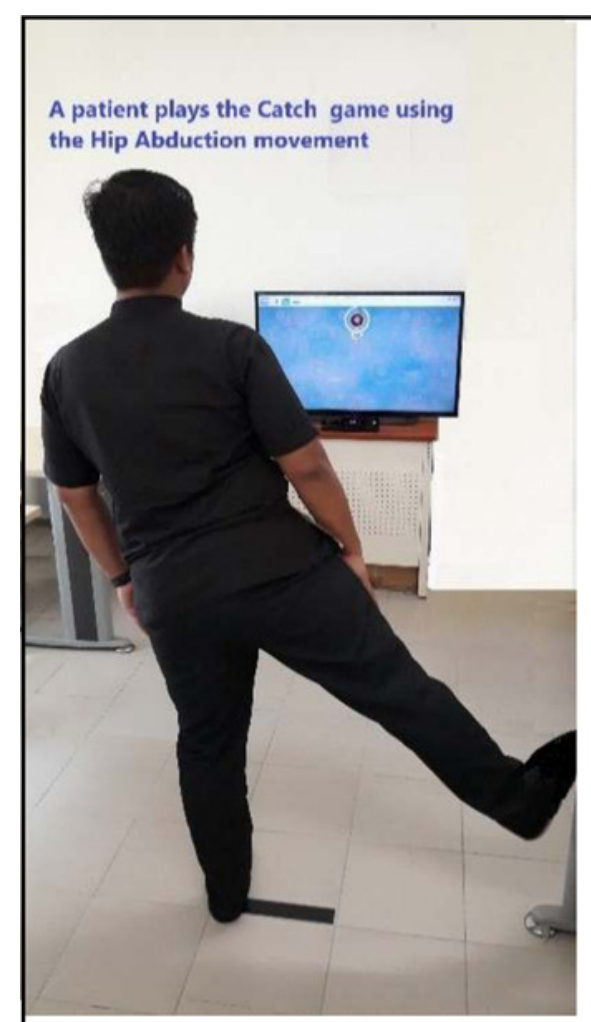

(a)

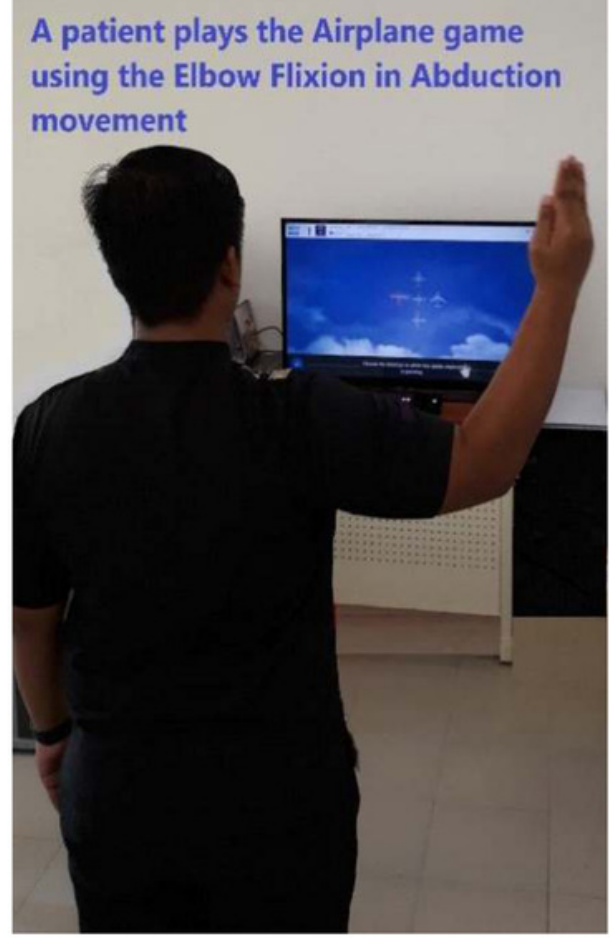

(b)

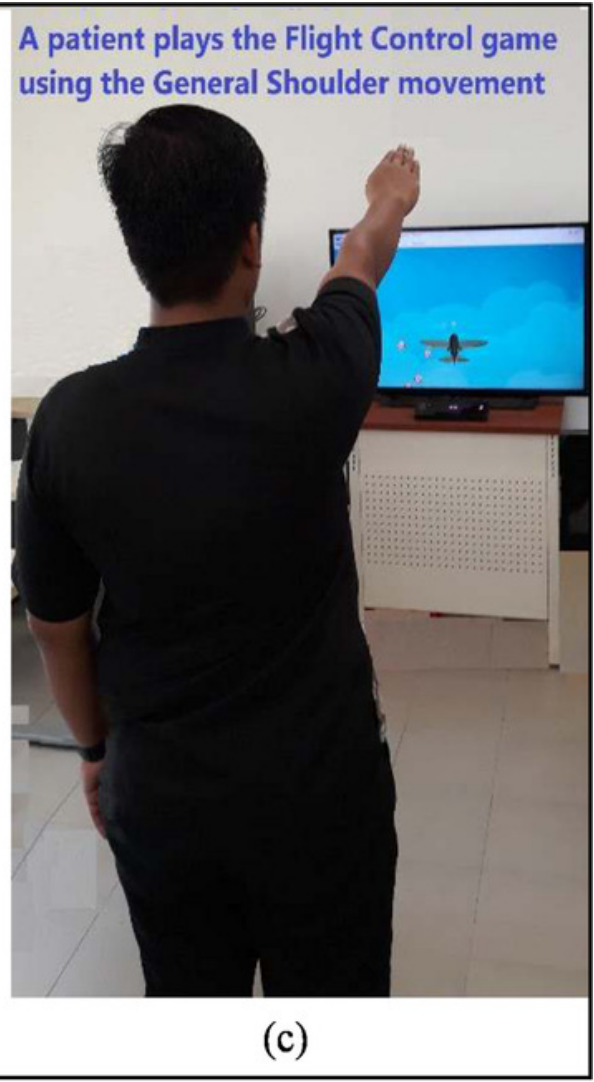


Figure 2

An example of the dialogue box for item settings.

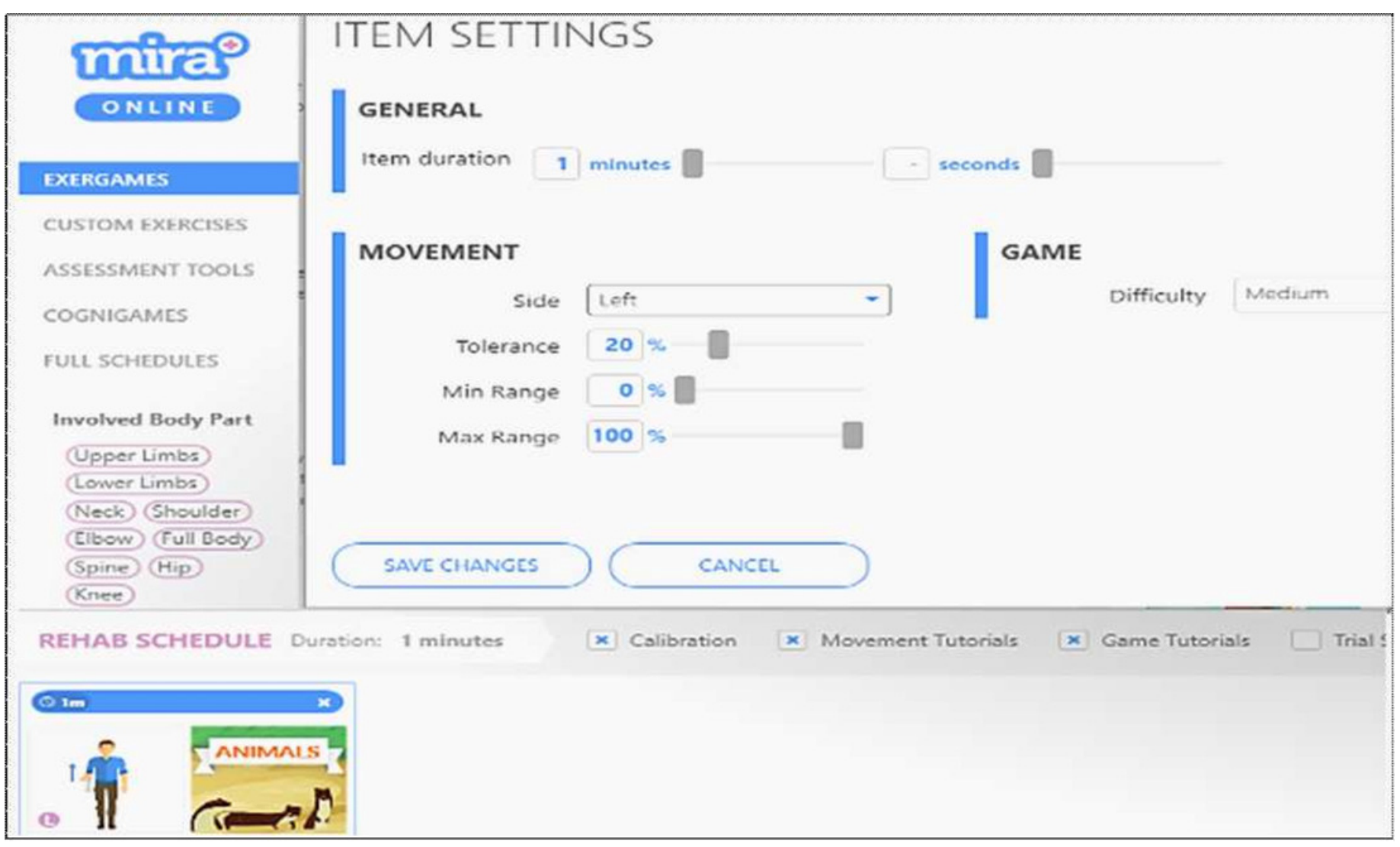


Figure 3

The framework of the ReComs approach.

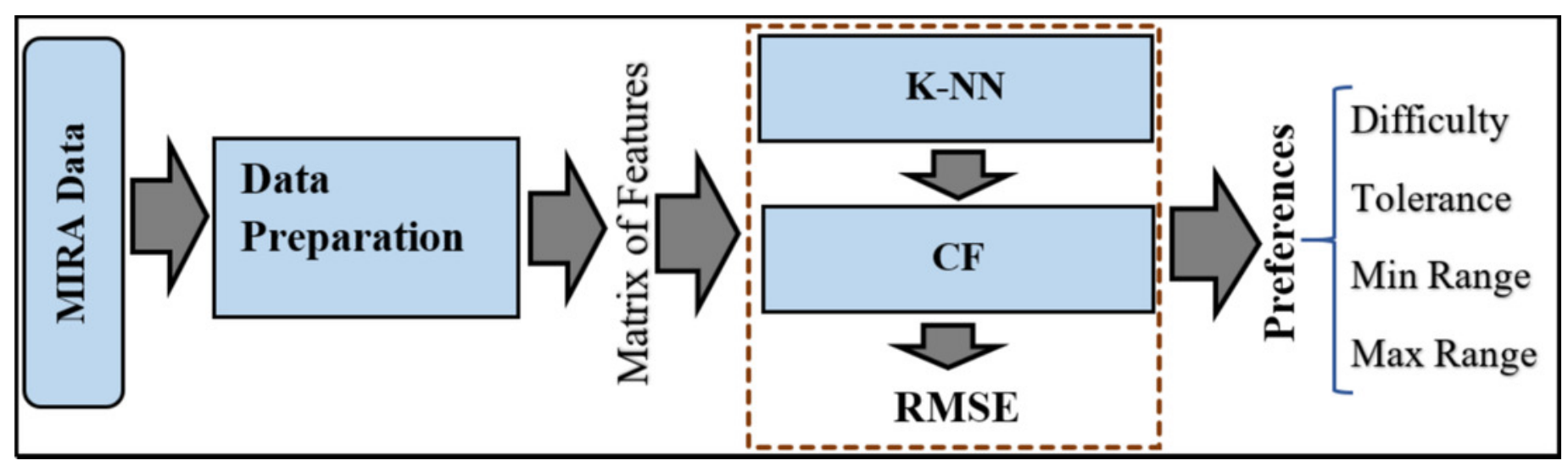


Figure 4

The framework of the ReComs+ approach.

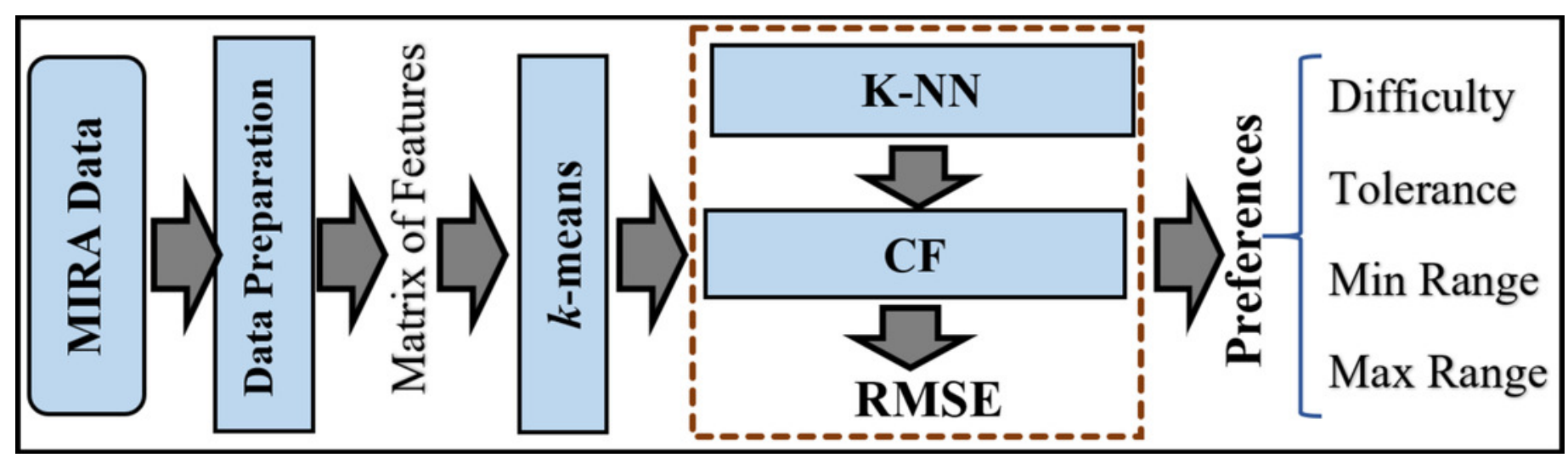


Figure 5

The framework of the ReComs++ approach.

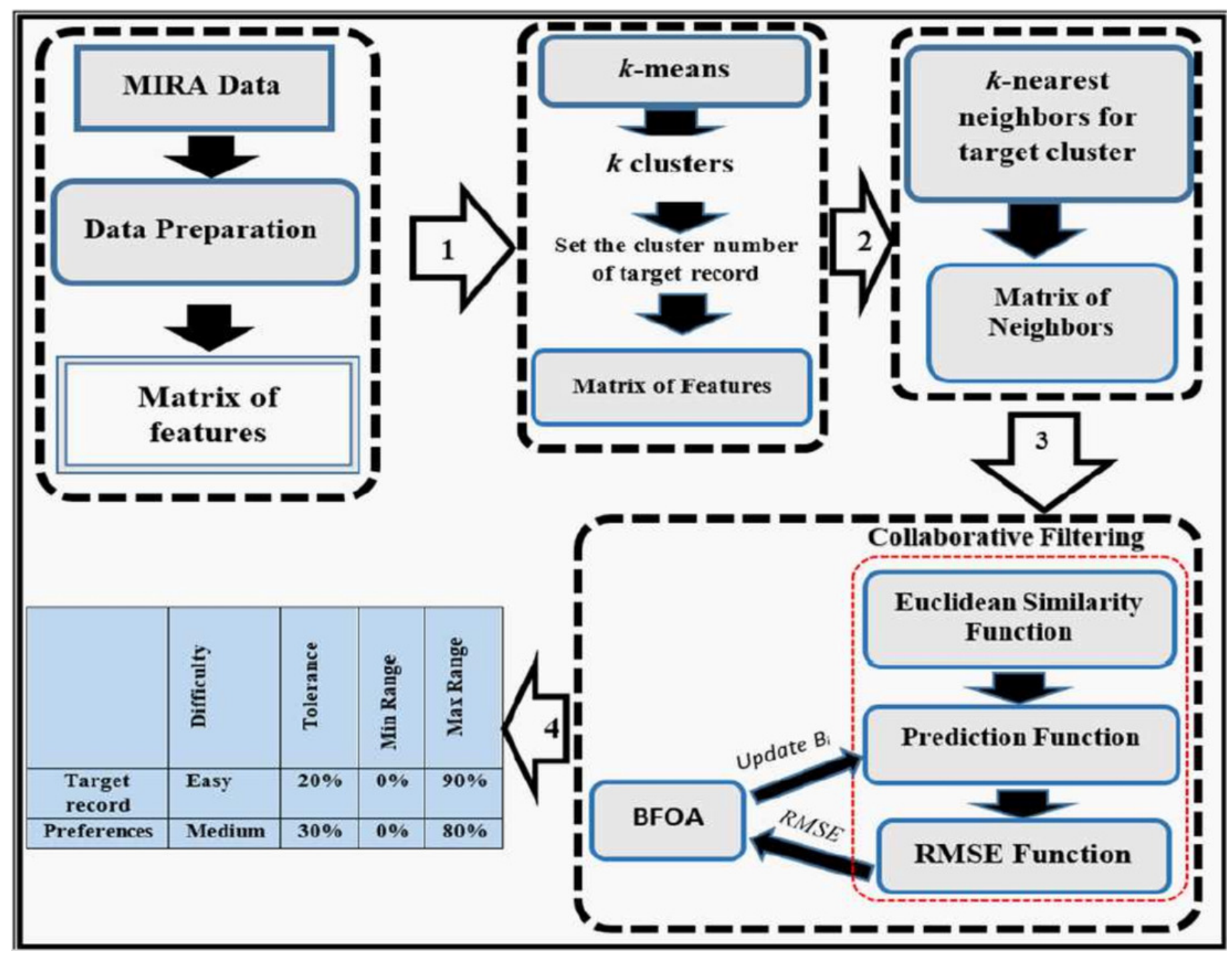


Figure 6

An example of the overfitted predicted features using ReComS.

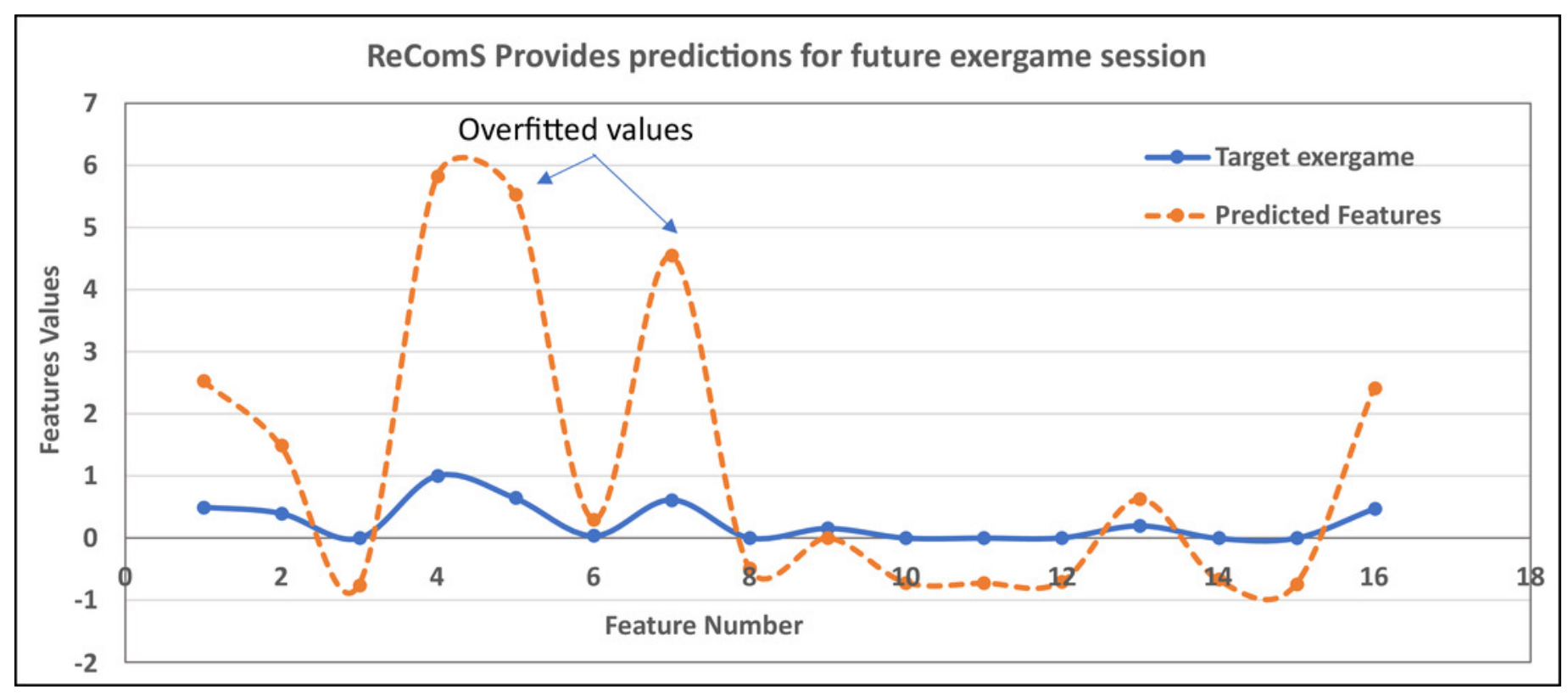


Figure 7

The prediction performance (RMSE) of ReComS.

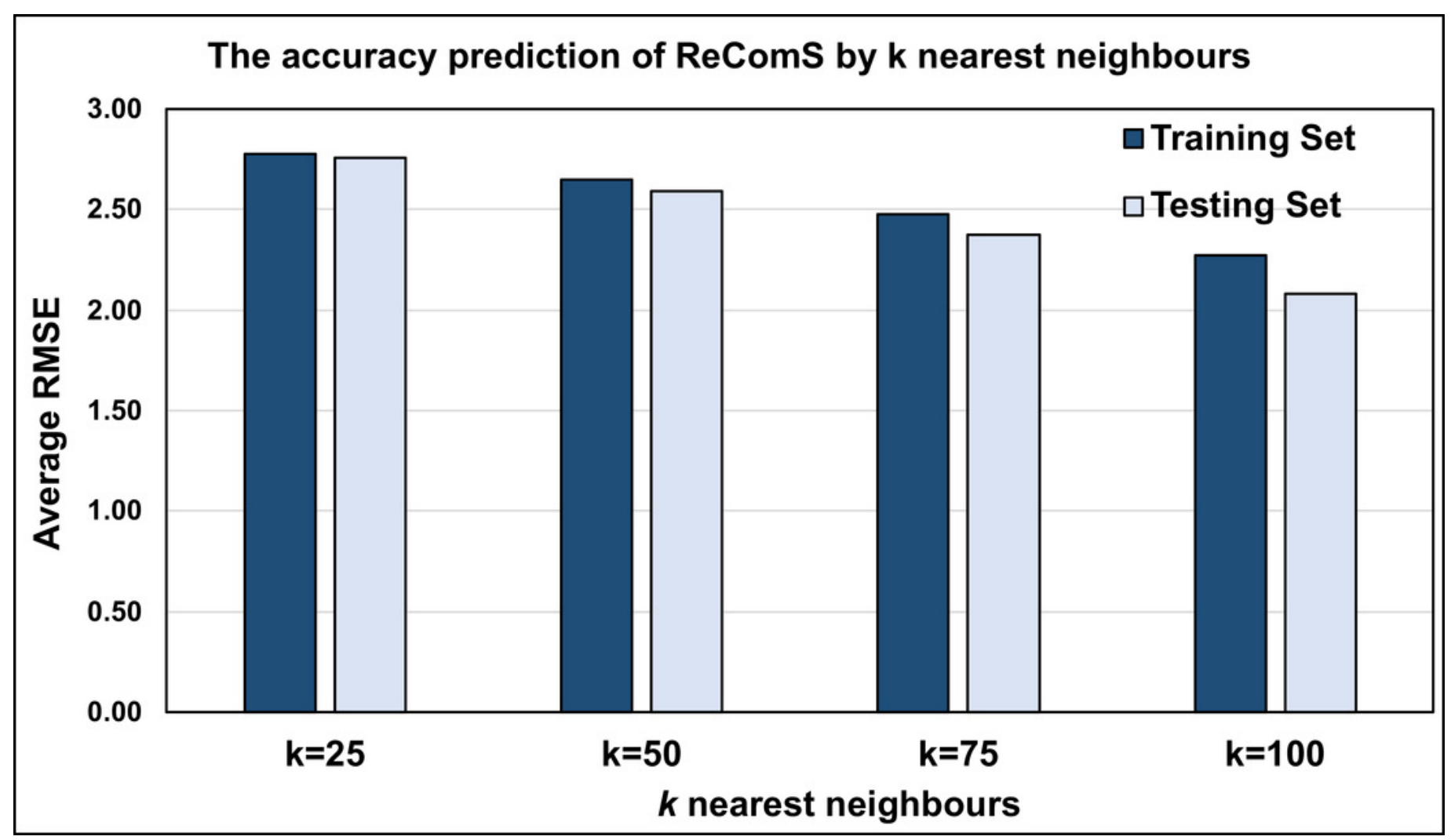


Figure 8

The prediction performance of ReComS+ using five clusters.

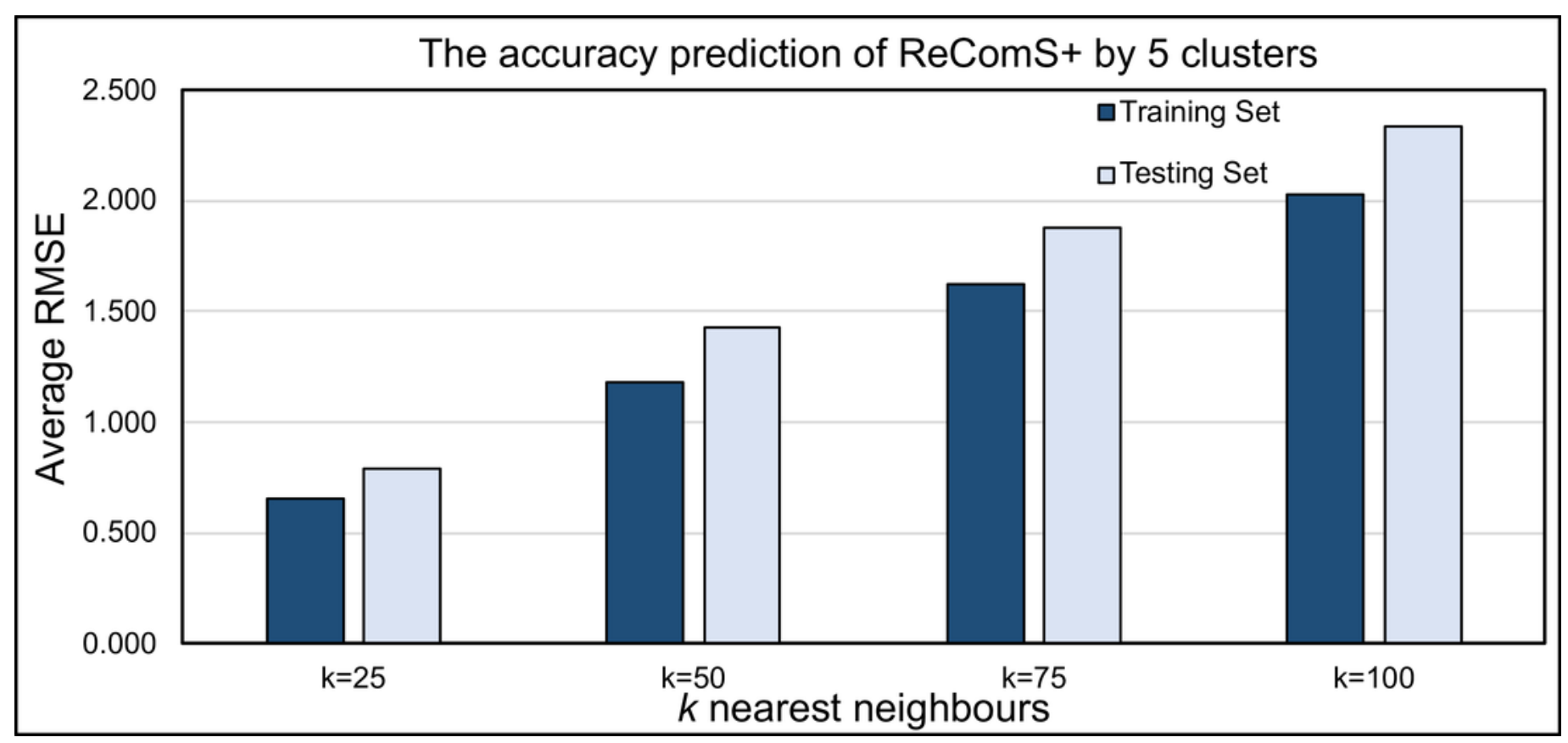


Figure 9

The prediction performance (RMSE) of ReComS+ using various clusters.

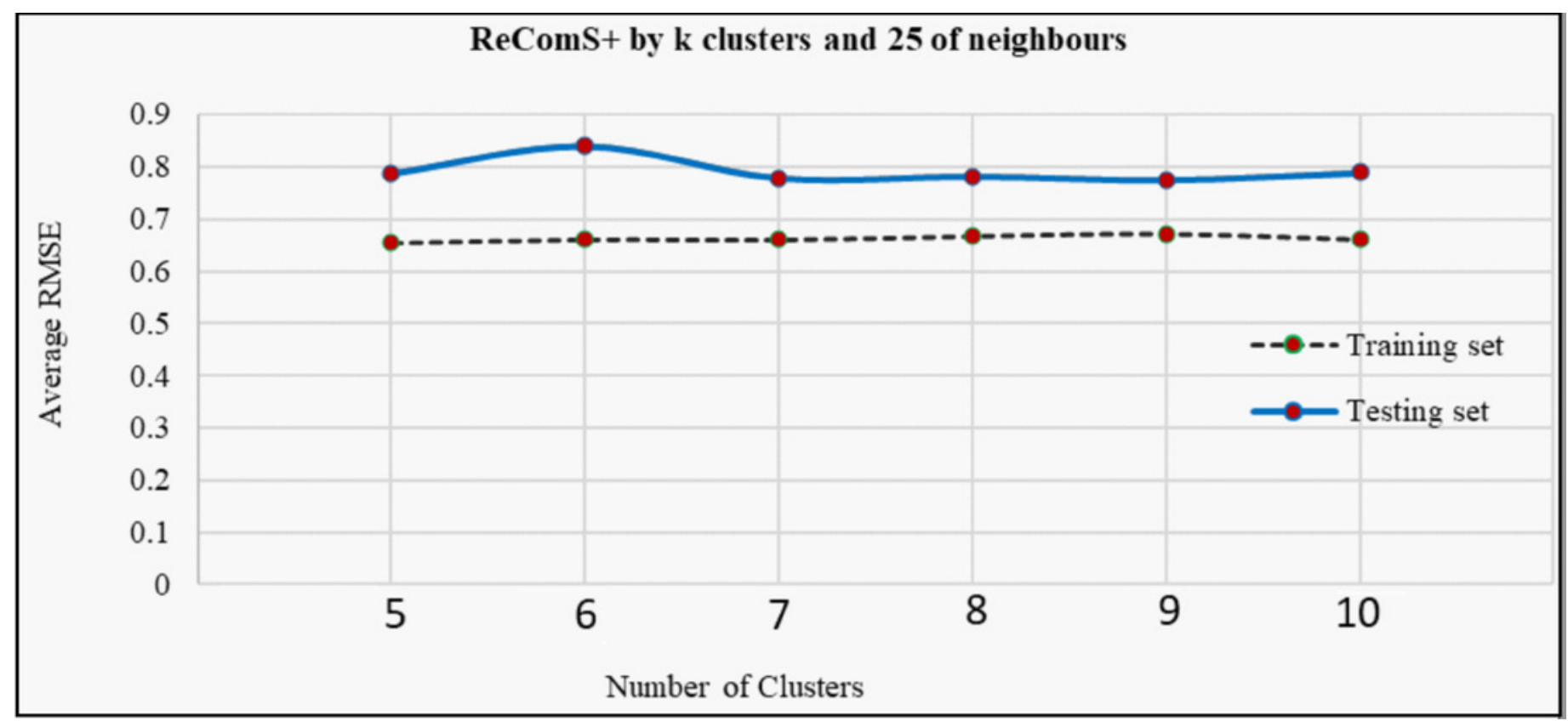


Figure 10

The prediction performance of the ReComS ++ approach based on optimisation.

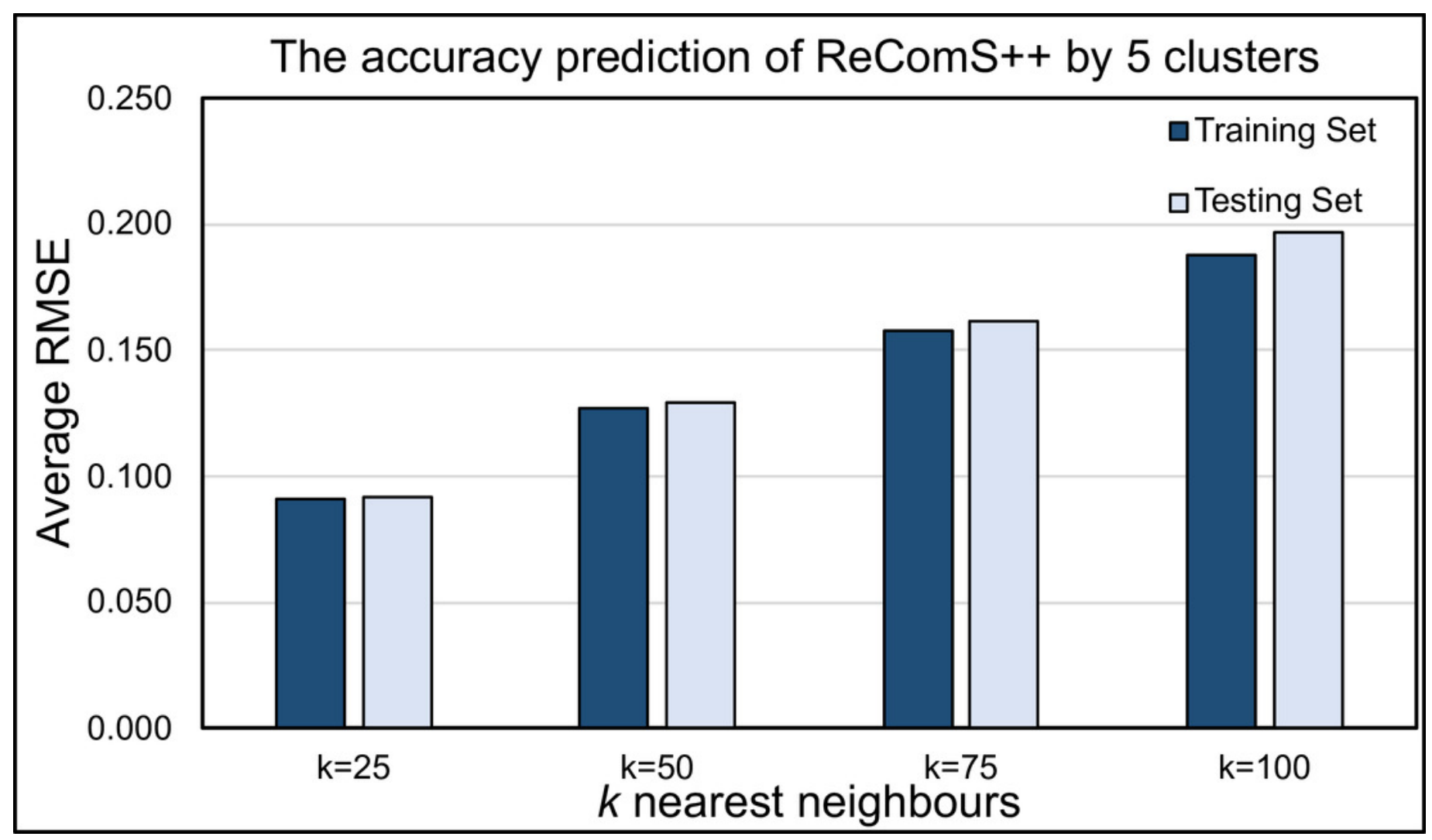


Figure 11

Comparisons among the ReComS approaches

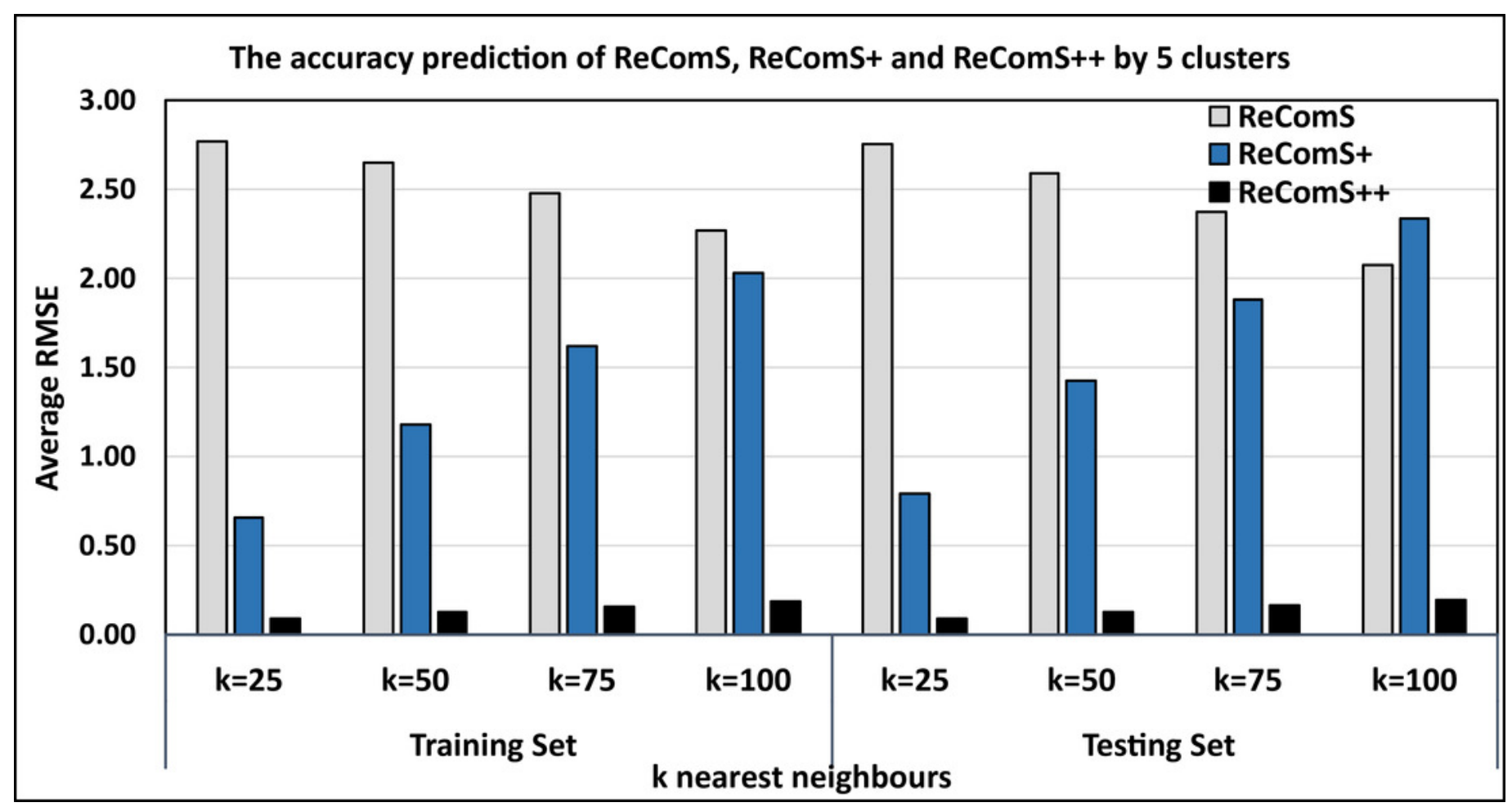


Figure 12

The threshold for deciding the predicted variables' values of the item setting in MIRA

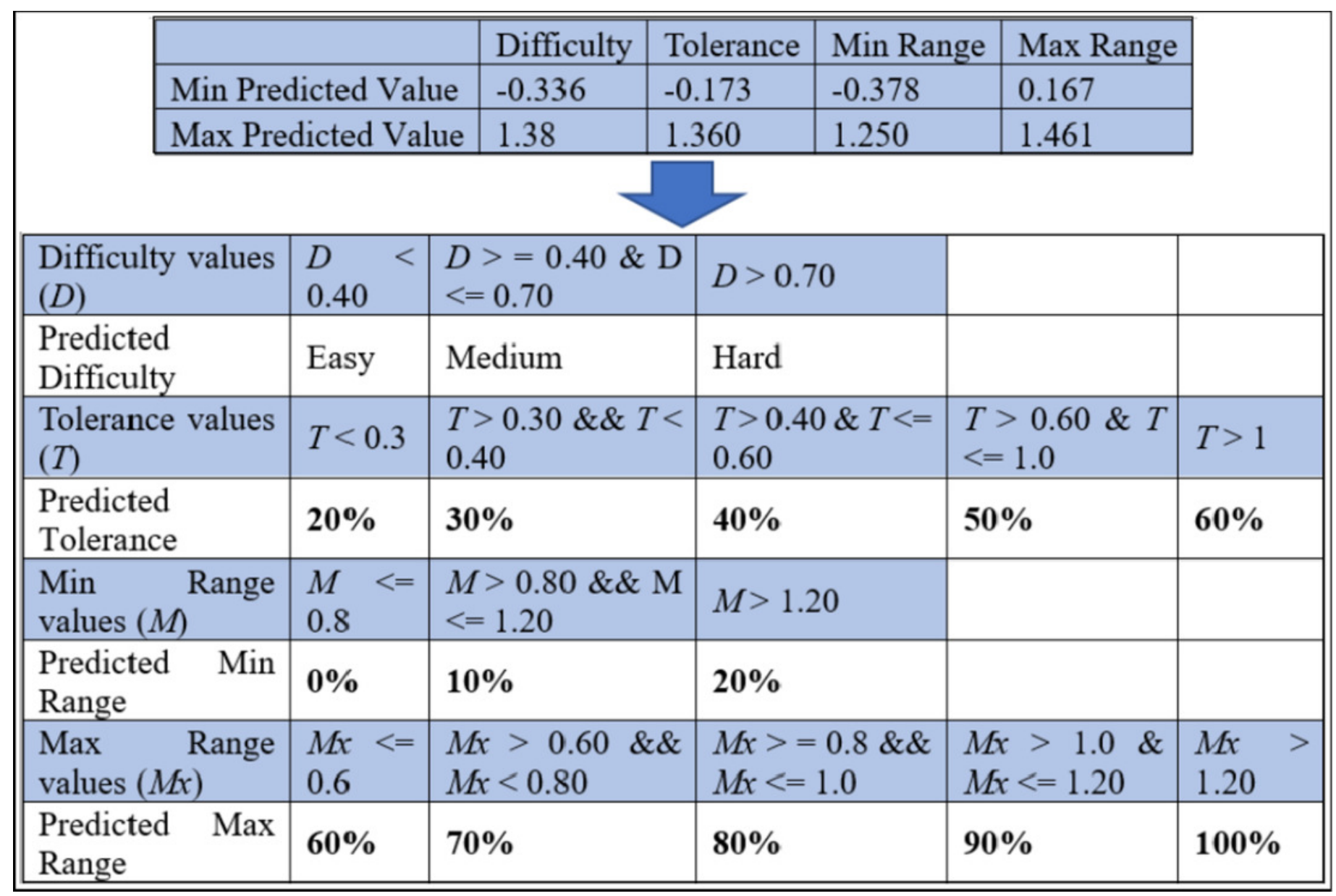


Figure 13

An example of the output predicted values for the item setting by the ReComs approach.

\begin{tabular}{||l|l|c|c|c|c||}
\hline \multirow{2}{*}{ MIRA Application } & Variables of item setting & Difficulty & Tolerance & $\begin{array}{c}\text { Min } \\
\text { Range }\end{array}$ & $\begin{array}{c}\text { Max } \\
\text { Range }\end{array}$ \\
\hline \multirow{2}{*}{ Target Record of item } & Actual values in data of MIRA & Easy & $30 \%$ & $0 \%$ & $60 \%$ \\
\cline { 2 - 6 } & Values by Normalisation & 0 & 0.3 & 0 & 0.6 \\
\hline \multirow{2}{*}{$\begin{array}{l}\text { Predicted Values of the } \\
\text { future item }\end{array}$} & Actual predicted values & -0.075 & 0.221 & -0.086 & 0.759 \\
\cline { 2 - 6 } & Predicted Values by Threshold & Easy & $20 \%$ & $0 \%$ & $70 \%$ \\
\hline \multirow{2}{*}{ Target Record of item } & Actual values in data of MIRA & Medium & $100 \%$ & $0 \%$ & $100 \%$ \\
\cline { 2 - 6 } & Values by Normalisation & 0.5 & 1 & 0 & 1 \\
\hline \multirow{2}{*}{$\begin{array}{l}\text { Predicted Values of the } \\
\text { future item }\end{array}$} & Actual predicted values & 0.823 & 1.035 & 0.188 & 0.897 \\
\cline { 2 - 6 } & Predicted Values by Threshold & Hard & $60 \%$ & $0 \%$ & $80 \%$ \\
\hline
\end{tabular}




\section{Figure 14}

MIRA interface provides preferences by ReComS ++ for the input settings.

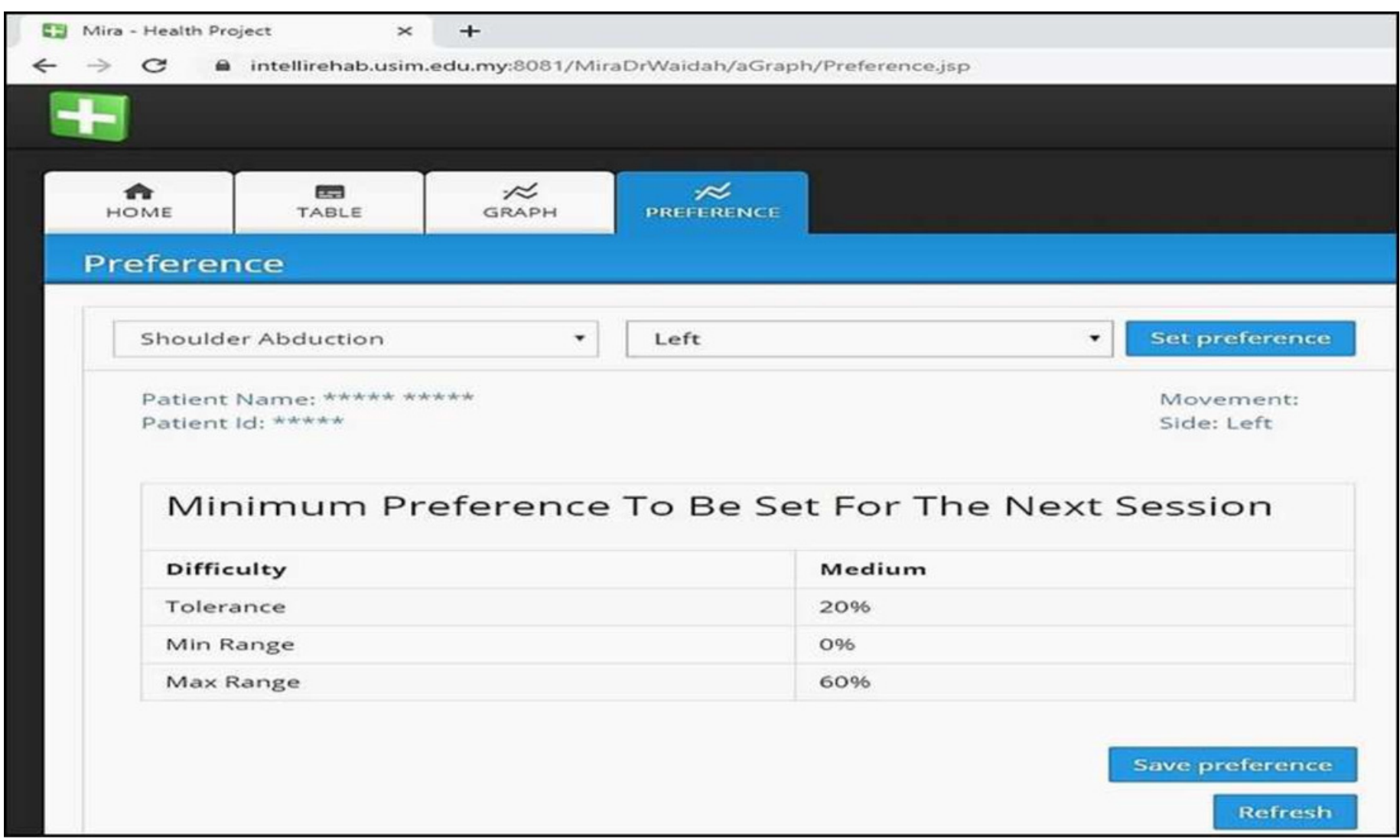




\section{Table $\mathbf{1}$ (on next page)}

Description of the output variables by the MIRA platform. 


\begin{tabular}{|c|c|c|}
\hline No & MIRA Application Data & Description \\
\hline 1 & Time (Duration) & Total time of the Exergame Item (a game and a movement). \\
\hline 2 & Still Time & $\begin{array}{l}\text { The idle time of the item when the patient stops moving before the } \\
\text { game finishes. }\end{array}$ \\
\hline 3 & Moving Time & $\begin{array}{l}\text { Time of the movement when the patient continues moving } \\
\text { correctly and incorrectly throughout a game-time. }\end{array}$ \\
\hline 4 & Moving Time in Exercise & $\begin{array}{l}\text { The time of the movement when the patient continues moving } \\
\text { correctly (as required by the exercise) throughout the exergame- } \\
\text { time. }\end{array}$ \\
\hline 5 & Average Acceleration & $\begin{array}{l}\text { The average of the positive change rate of the velocity divided by } \\
\text { the overall duration of the exergame. }\end{array}$ \\
\hline 6 & Average deceleration & $\begin{array}{l}\text { The average of the negative change rate of the velocity divided by } \\
\text { the overall duration of the exergame. }\end{array}$ \\
\hline 7 & Average Accuracy & The accuracy of the movement for each exergame. \\
\hline 8 & $\begin{array}{l}\text { Average congruent correct } \\
\text { answer reaction time }\end{array}$ & $\begin{array}{l}\text { The movement with the objects in the game during positive } \\
\text { reaction time where the reaction time is the positive response } \\
\text { time for each event in the game. }\end{array}$ \\
\hline 9 & $\begin{array}{l}\text { Average congruent } \\
\text { incorrect answer reaction } \\
\text { time }\end{array}$ & $\begin{array}{l}\text { Congruent movement with the objects during a negative reaction } \\
\text { time by responding to each event. }\end{array}$ \\
\hline 10 & Average percentage & $\begin{array}{l}\text { Average Range of motion that the patient performs during the } \\
\text { exercise. }\end{array}$ \\
\hline 11 & Average speed & $\begin{array}{l}\text { The result by dividing the Distance with the overall Time duration } \\
\text { when the movement is performed correctly. }\end{array}$ \\
\hline 12 & Average variation & $\begin{array}{l}\text { The average interval of Range of Motion, a patient, performs } \\
\text { during an exercise. }\end{array}$ \\
\hline 13 & Distance & Total distances performed by a specific joint. \\
\hline 14 & Maximum percentage & $\begin{array}{l}\text { Maximum Range of Motion carried out by a patient throughout the } \\
\text { exergame. }\end{array}$ \\
\hline 15 & Minimum percentage & $\begin{array}{l}\text { Minimum Range of Motion carried out by a patient throughout the } \\
\text { exergame. }\end{array}$ \\
\hline 16 & Repetition & The total number of correct movements throughout the exergame. \\
\hline 17 & Points & The total scores achieved throughout the exergame. \\
\hline
\end{tabular}




\section{Table 2 (on next page)}

Description of MIRA platform settings. 


\begin{tabular}{|c|c|c|}
\hline No & $\begin{array}{l}\text { MIRA platform } \\
\text { setting }\end{array}$ & Description \\
\hline 1 & Item Duration & $\begin{array}{l}\text { Assign the duration of the exergame according to the patient's ability between } 1 \\
\text { and } 10 \text { minutes ( } 1 \text { minute is the default duration). }\end{array}$ \\
\hline 2 & Side & $\begin{array}{l}\text { Assign the side of the patient used in the exergame, which can be Left or Right, or } \\
\text { even Both. }\end{array}$ \\
\hline 3 & Tolerance & $\begin{array}{l}\text { Assign the percentage between } 0 \% \text { and } 100 \% \text {. The tolerance is the accepted error } \\
\text { in performing the required exercise, to enable the patient to play easily when they } \\
\text { cannot perform the entire correct movement of the exercise. }\end{array}$ \\
\hline 4 & Min Range & $\begin{array}{l}\text { Assign the percentage between } 0 \% \text { and } 100 \% \text {. The minimum Range of Motion } \\
\text { required by the patient to play exergames. }\end{array}$ \\
\hline 5 & Max Range & $\begin{array}{l}\text { Assign the percentage between } 0 \% \text { and } 100 \% \text {. The maximum Range of Motion } \\
\text { required by the patient who plays an exergame. }\end{array}$ \\
\hline 6 & $\begin{array}{l}\text { Difficulty } \\
\text { Game }\end{array}$ & Assign the difficulty of the exergame with values Easy, Medium or Hard. \\
\hline
\end{tabular}




\section{Table 3(on next page)}

The factors' values of BFOA. 


\begin{tabular}{llll}
\hline BFOA factors & No & Parameters & No \\
\hline P dimension of search space & 30 & Reproduction steps & 4 \\
Number of bacteria groups $S$ & 6 & Elimination-dispersal steps & 4 \\
Number of iterations & 20 & Probability of elimination-dispersal & 0.25 \\
Optimum RMSE & 0.1 & $d_{\text {attract }}$ & 0.1 \\
Run length unit $C_{i}$ & 0.09 & $w_{\text {attract }}$ & 0.2 \\
chemotactic steps & 6 & $h_{\text {repellant }}$ & 0.1 \\
The swimming length & 4 & $w_{\text {repellant }}$ & 5 \\
\hline
\end{tabular}

1 


\section{Table 4 (on next page)}

An example of information collected by the physiotherapist for MIRA and ReComS ++ . 


\begin{tabular}{|c|c|c|c|c|c|c|c|c|c|}
\hline \multirow[b]{2}{*}{ Date } & \multirow[b]{2}{*}{ 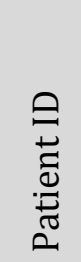 } & \multirow[b]{2}{*}{ Movement } & \multirow[b]{2}{*}{$\frac{0}{0}$} & \multicolumn{4}{|c|}{ Preference } & \multirow[b]{2}{*}{ Game } & \multirow[b]{2}{*}{ 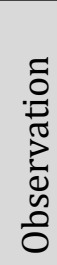 } \\
\hline & & & & $\begin{array}{l}\stackrel{U}{0} \\
\tilde{\Xi} \\
0 \\
0 \\
0\end{array}$ & $\begin{array}{l}\mathscr{\Xi} \\
\mathbb{\pi} \\
\approx \\
\Xi \\
\Sigma\end{array}$ & 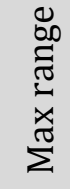 & 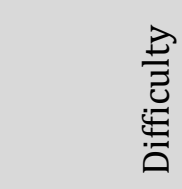 & & \\
\hline \multirow[t]{4}{*}{$5 / 8 / 2$} & 352 & Elbow Flexion & $\mathrm{R}$ & 2 & 0 & 10 & Easy & Catch & $\mathrm{P}$ \\
\hline & & General Arm & $\mathrm{R}$ & 2 & 0 & 80 & Easy & Catch & $\mathrm{P}$ \\
\hline & & Shoulder Internal & $\mathrm{R}$ & 2 & 0 & 70 & Easy & Catch & $\mathrm{P}$ \\
\hline & & Shoulder External & $\mathrm{R}$ & 2 & 0 & 10 & Easy & Catch & $\mathrm{P}$ \\
\hline \multirow[t]{3}{*}{$5 / 8 / 2$} & 356 & Elbow Flexion with & $\mathrm{L}$ & 3 & 0 & 10 & Easy & Colour & $\mathrm{P}$ \\
\hline & & General Full Body & $\mathrm{L}$ & 2 & 0 & 10 & Easy & Basketball & $\mathrm{P}$ \\
\hline & & Shoulder Abduction & $\mathrm{L}$ & 2 & 0 & 10 & Easy & Colour & $\mathrm{P}$ \\
\hline \multirow[t]{4}{*}{$5 / 8 / 2$} & 359 & Elbow Flexion & $\mathrm{L}$ & 2 & 0 & 10 & Medium & Catch & $\mathrm{N}$ \\
\hline & & Spine Lateral Flexion & $\mathrm{L}$ & 2 & 0 & 10 & Medium & Colour & $\mathrm{P}$ \\
\hline & & Spine Frontal Flexion & $\mathrm{L}$ & 2 & 0 & 10 & Medium & Catch & $\mathrm{P}$ \\
\hline & & Shoulder Internal & $\mathrm{L}$ & 2 & 0 & 10 & Medium & Catch & $\mathrm{P}$ \\
\hline \multirow[t]{6}{*}{$5 / 8 / 2$} & 355 & Elbow Flexion & $\mathrm{L}$ & 3 & 0 & 10 & Easy & Catch & $\mathrm{P}$ \\
\hline & & Spine Frontal Flexion & $\mathrm{L}$ & 2 & 0 & 70 & Easy & Catch & $\mathrm{P}$ \\
\hline & & Spine Lateral Flexion & $\mathrm{L}$ & 3 & 0 & 10 & Easy & Colour & $\mathrm{P}$ \\
\hline & & Functional Reach & $\mathrm{L}$ & 3 & 0 & 70 & Easy & Grab & $\mathrm{P}$ \\
\hline & & Shoulder Frontal Flexion & $\mathrm{L}$ & 3 & 0 & 10 & Easy & Colour & $\mathrm{P}$ \\
\hline & & Sit To Stand & $\mathrm{L}$ & 2 & 0 & 10 & Medium & Atlantis & $\mathrm{P}$ \\
\hline \multirow[t]{3}{*}{$5 / 8 / 2$} & 359 & Elbow Flexion in & $\mathrm{L}$ & 2 & 0 & 10 & Medium & Colour & $\mathrm{P}$ \\
\hline & & General Full Body & $\mathrm{L}$ & 2 & 0 & 10 & Medium & Basketball & $\mathrm{P}$ \\
\hline & & Functional Reach & $\mathrm{L}$ & 2 & 0 & 10 & Medium & Grab & $\mathrm{P}$ \\
\hline \multirow[t]{4}{*}{$6 / 8 / 2$} & 350 & General Arm & $\mathrm{R}$ & 2 & 0 & 10 & Easy & Catch & $\mathrm{P}$ \\
\hline & & General Shoulder & $\mathrm{R}$ & 2 & 0 & 10 & Medium & Catch & $\mathrm{P}$ \\
\hline & & Elbow Flexion & $\mathrm{L}$ & 2 & 0 & 10 & Easy & Catch & $\mathrm{N}$ \\
\hline & & Spine Lateral Flexion & & 3 & 0 & 10 & Easy & Colour & $\mathrm{P}$ \\
\hline \multirow[t]{4}{*}{$6 / 8 / 20$} & 354 & Elbow Flexion & $\mathrm{L}$ & 2 & 0 & 10 & Medium & Catch & $\mathrm{P}$ \\
\hline & & Shoulder Internal & $\mathrm{L}$ & 2 & 0 & 10 & Medium & Catch & $\mathrm{P}$ \\
\hline & & General Arm & $\mathrm{L}$ & 2 & 0 & 10 & Medium & Catch & $\mathrm{P}$ \\
\hline & & General Arm & $\mathrm{R}$ & 2 & 0 & 10 & Medium & Catch & $\mathrm{P}$ \\
\hline
\end{tabular}




\section{Table 5 (on next page)}

Effectiveness of preferences by ReComS ++ according to the physiotherapist's observation. 


\begin{tabular}{lcc}
\hline Positive Preferences & Number & Results by Percentage \\
\hline Patients have played a set of Exergames by MIRA & 28 & \\
Period of Evaluation & 5 weeks & \\
Exergames Preferences & 1182 & \\
Negative Preferences (N) & 168 & $14.21 \%$ \\
Positive Preferences (P) & 1014 & $85.79 \%$ \\
\hline
\end{tabular}

1

2 